\title{
LINE-1 and Alu methylation signatures in autism spectrum disorder and their function in the regulation of autism-related genes
}

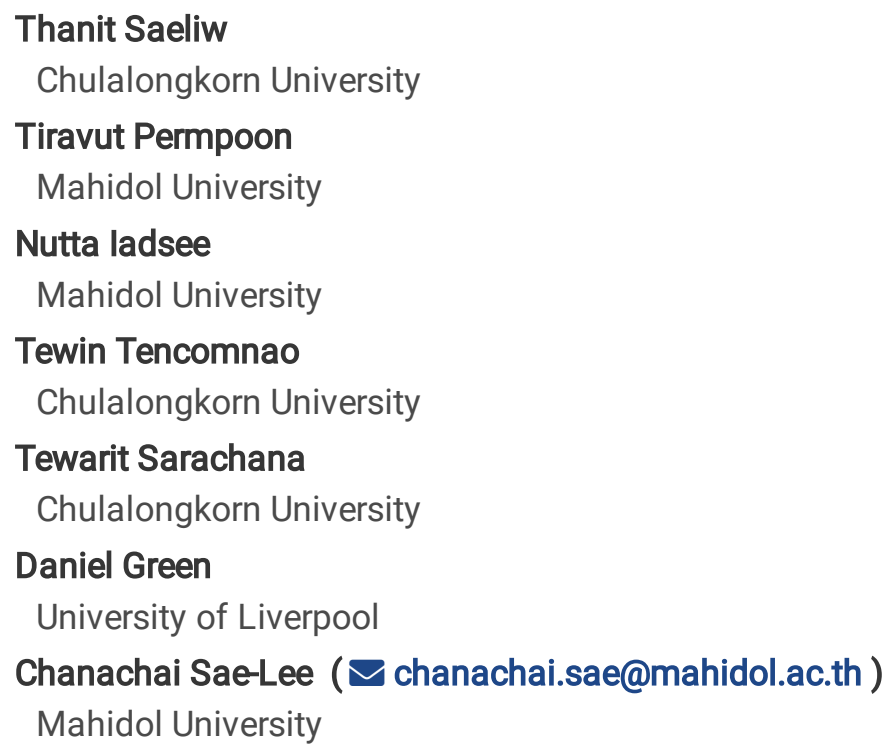

\section{Research Article}

Keywords: Autism Spectrum Disorder, DNA methylation, LINE-1, Alu element, Epigenetic age

Posted Date: February 16th, 2022

DOI: https://doi.org/10.21203/rs.3.rs-1194570/v2

License: (a) (1) This work is licensed under a Creative Commons Attribution 4.0 International License. Read Full License 


\section{Abstract}

\section{Background}

Long interspersed nucleotide element-1 (LINE-1) and Alu elements are retrotransposons whose abilities cause abnormal gene expression and genomic instability. Several studies have focused on DNA methylation profiling of gene regions, but the locusspecific methylation of LINE-1 and Alu elements has not been identified in autism spectrum disorder (ASD).

\section{Methods}

DNA methylation age was predicted using Horvath's method. We interrogated locus- and family-specific methylation profiles of LINE-1 and Alu elements (22,352 loci) in ASD blood using publicly-available Illumina Infinium 450K methylation datasets from heterogeneous ASD ( $\mathrm{n}=52)$, ASD with 16p11.2 del $(\mathrm{n}=7)$, and ASD with Chromodomain Helicase DNA-binding 8 $(C H D 8)$ variants $(n=15)$. The differentially methylated positions of LINE-1 and Alu elements corresponding to genes were combined with transcriptome data from multiple ASD studies.

\section{Results}

We identified the epigenetic age acceleration significantly decelerated in ASD children over the age of 11 years. We further interrogated locus- and family-specific methylation profiles of LINE-1 and Alu elements (22,352 loci) in ASD blood using publicly-available Illumina Infinium 450K methylation datasets from heterogeneous ASD $(n=52)$, ASD with 16p11.2 del $(n=$ $7)$, and ASD with Chromodomain Helicase DNA-binding $8(C H D 8)$ variants $(n=15)$. DNA methylation profiling revealed LINE-1 and Alu methylation signatures in each ASD risk loci by which global methylation were notably hypomethylated exclusively in ASD with CHD8 variants. When LINE-1 and Alu elements were clustered into subfamilies, we found methylation changes in a family-specific manner in L1P, L1H, HAL, AluJ, and AluS families in the heterogeneous ASD and ASD with CHD8 variants. Interesting, our results showed that LINE-1 and Alu methylation within target genes is inversely related to the expression level in each ASD variant. Finally, we demonstrate the potential for LINE-1 and Alu methylation signatures to predict ASD individuals from non-ASD.

\section{Conclusions}

The DNA methylation signatures of the LINE-1 and Alu elements in ASD, as well as their functional impact on ASD-related genes, have been identified. If confirmed in future larger studies, these finding may contribute to the identification of epigenomic biomarkers of ASD in those with high risk of ASD.

\section{Background}

Autism spectrum disorder (ASD) is a complex neurodevelopmental disorder characterized by two behavioral impairments: (i) deficits in social interactions and communication, and (ii) restricted interests and repetitive behaviors, according to the Diagnostic and Statistical Manual of Mental Disorders, Fifth Edition criteria (1). According to the Centers for Disease Control and Prevention (CDC), the prevalence of ASD has risen dramatically over the last decade due to better screening methods (2). In 2016, ASD affected approximately one out of every 54 children in the United States (2). ASD is currently understood as a multifactorial disorder, with the precise causes remaining unknown. Over the last two decades, research has attempted to elucidate the genetic origin of the disorder. However, genetic aberration is only found in $10-20 \%$ of ASD cases. In total, more than $60 \%$ of people with ASD are idiopathic (3). Several studies have shown that ASD clinical phenotypic heterogeneity is influenced by a combination of genetic and environmental factors (4-6). This evidence has highlighted non-genetic factors such as epigenetics (DNA methylation (DNAm)) and environmental interactions as key players in ASD progression. Additionally, some genetic factors that increase the risk of ASD, but only a few loci have a high impact on ASD (7). The 16p11.2 deletion (16p11.2 del) and Chromodomain helicase DNA-binding domain 8 (CHD8) variants are high genetic risk factors for ASD $(8,9)$. People with 16p11.2del are usually characterized by developmental delay, intellectual disability, or 
ASD (9). CHD8 is strongly associated with ASD and other neurodevelopmental disorders including schizophrenia and intellectual disability $(2,10)$.

Epigenetics is a family of heritable mechanisms that elicit control of gene expression without modification to DNA sequences (11). Examples of epigenetic mechanisms are DNAm, RNA modification, and histone modifications (12). DNAm, the most frequently studied epigenetic modification, involves the addition of methyl groups to DNA. Depending on its genomic location, the addition of a methyl groups to the $5^{\text {th }}$ carbon atom of cytosine can have repressive or inductive effects on the gene expression. When DNAm is not properly maintained or established, methylation abnormalities can manifest in disease development. DNAm patterns are well known to show tissue-specific differentially methylated regions (DMRs). However, most loci present similar DNAm levels across a wide variety of tissue types. Interestingly, recent work has demonstrated the utility of blood as a surrogate for human brain tissue $\mathrm{CpG}$ methylation (13). Therefore, blood-based epigenetic biomarkers have the potential to serve as non-invasive biomarkers for otherwise inaccessible tissues. For instance, some epigenetic markers in blood have been identified as biomarkers in early stages of Alzheimer's disease (14). Similar findings have also arisen in ASD, a recent meta-analysis of blood-based DNA demonstrated evidence of the associations between blood-based and brain samples in comparison between ASD and controls (15).

Long interspersed nucleotide element-1 (LINE-1) and Alu elements are known as non-long terminal repeat retrotransposons that can replicate and insert themselves into different locations within the host genome. LINE-1 and Alu elements make up more than $25 \%$ of the human genome and have a copy number of over one million elements (16). These repetitive elements (REs) can affect the expression of host or neighboring protein-coding genes through introducing alternative promoters or enhancers, novel splicing sites, and epigenetic alteration through DNAm (16). Subfamilies of LINE-1 and Alu elements can be subcategorized by identifying variants in their sequences that have accumulated in the evolutionary heritage $(17,18)$. LINE-1 has been classified into three main subfamilies during early primate evolution including L1M (mammalian-specific, oldest), L1P (primate-specific, intermediate), and L1H (human-specific, youngest) subfamilies (19). Alu elements have been classified into three main subfamilies including AluJ (oldest), AluS (intermediate), and AluY (youngest) (17). The ability to transposition has been lost in the oldest subfamilies of both LINE-1 and Alu, whereas the intermediate and young subfamilies (L1PA, L1H, AluS, and AluY) are active and capable of jumping (20).

Current evidence suggests that aberrant DNAm of LINE-1 and Alu elements links to several diseases: ASD (21), presymptomatic dementia in type 2 diabetes (22), and chronic lymphocytic leukemia (23). Whole-genome sequencing investigation of the brains of individuals with ASD revealed that LINE-1 and Alu elements have a larger number of insertions than in normal brain tissues (24). The binding of methyl-CpG binding protein 2 (MeCp2), transcriptional repressor, to the LINE1 promoter was dramatically reduced, and this was related to LINE-1 overexpression in ASD brains (25). The functional impact of LINE-1 and Alu elements in the ASD is currently unknown. One possibility is that LINE-1 and Alu elements act as enhancers or alternative promoters for host genes. Our recent study discovered a link between LINE-1/Alu elements and gene expression in blood transcriptome, implying that LINE-1 and Alu may influence the expression of host genes in ASD $(21,26)$. Additionally, we also found changes in global methylation of LINE-1 and Alu elements in the lymphoblastoid cell line of the ASD subgroup based on clinical phenotypes $(21,26)$. According to a recent study using blood samples from ASD, one of CpG sites within the LINE-1 sequence showed a slight decrease of methylation levels in ASD compared to unaffected controls but its methylation level was highly significant in ASD with mental regression (27). However, locus- and family-specific methylation patterns of LINE-1 and Alu elements in ASD blood have not been reported.

Here, we intended to investigate the DNAm profile of LINE-1 and Alu elements, as well as the functional impact on regulation genes located nearby these elements. Using Illumina Infinium 450K annotation, CpG sites mapping to LINE-1 and Alu families were identified from DNAm data (GSE113967 and GSE131706) obtained from the NCBI Gene Expression Omnibus (GEO) database. We calculated DNAm Age in ASD blood using Horvath's method. Differential methylation of LINE-1 and Alu elements was examined in a global, locus- and subfamily-specific manner for each ASD variant, including heterogeneous ASD ( $n=52)$, ASD with 16p11.2 del $(n=7)$, and ASD with CHD8 variants $(n=15)$. Biological functions and interactome networks of genes located nearby LINE-1 and Alu elements were predicted by ingenuity pathway analysis (IPA). We 
subsequently identified these genes that were reproducibly differentially expressed in transcriptome data obtained from multiple ASD cohorts. Finally, we used logistic regression to assess the accuracy of a unique LINE-1 and Alu methylation signatures in discriminating ASD with a genetic variant from controls.

\section{Methods}

\section{Data collection}

Differentially methylated retrotransposon loci were identified in publicly available Illumina Infinium 450K datasets through GEO DataSets: http://www.ncbi.nlm.nih.gov/gds (69): GSE113967 (63). In this dataset, ethical approval was granted by the Research Ethics Boards of the respective institutions (University of Michigan SickKids, Holland Bloorview Kids Rehabilitation Hospital, Western University, McMaster University) (63). Data were collected from the heterogeneous ASD ( $n=52)$, ASD with confirmed typical $600 \mathrm{Kbp}$ deletion in 16p11.2 del $(n=7)$, ASD with confirmed de novo CHD8 sequence variants $(n=15)$, and age-matched controls (non-ASD) $(n=48)$ (Table 1). Validation was performed in a cohort of genome-wide DNAm profiling of post-mortem brain tissue in the subventricular zone of the lateral ventricles from five individuals with ASD and five without (GSE131706) (33) (Table 1).

Genes with differently methylated loci were analyzed in publicly available gene expression datasets from publicly available datasets accessed via GEO using the following inclusion criteria: (1) the study must include ASD cases and controls; (2) the study must use microarray/RNA-seq technology; and (3) the study must use blood or post-mortem brain tissues. Finally, we obtained seven ASD studies, four of which used blood and three of which used post-mortem brain tissues (see Additional file $1)$.

Table 1 Characteristics of the Infinium450K datasets in the present study including ASD and non-ASD. The numbers of patients for each group are given for each gender. The ages for each group are given as means \pm standard deviation.

\begin{tabular}{lccc} 
Groups & \multicolumn{2}{c}{ Gender } & \multicolumn{1}{l}{ Age } \\
\cline { 2 - 3 } & Male & Female & \\
\cline { 1 - 2 } GSE113967: Blood & & & \\
\hline ASD & 45 & 7 & $10.45 \pm 3.31$ \\
\hline Non-ASD & 33 & 15 & $10.22 \pm 3.95$ \\
\hline ASD+CHD8 & 11 & 4 & $8.97 \pm 4.11$ \\
\hline ASD+16p11.2 del & 6 & 1 & $6.78 \pm 4.05$ \\
\hline GSE131706: Subventricular zone of the lateral ventricles \\
\hline ASD & 5 & - & $7.07 \pm 1.78$ \\
\hline Non-ASD & 5 & - & $7.70 \pm 1.40$
\end{tabular}

\section{Epigenetic clock}

DNAm Age was predicted using Horvath's method, which is available at methylclock package through the DNAmAge function (70). Horvath's method employed $353 \mathrm{CpG}$ sites with corresponding coefficient values to define DNAm Age as predicted age, in years (28). Age acceleration residuals were obtained from a multivariate model regression of DNAm Age on chronological age and estimates of blood cell counts. Age acceleration residuals were assessed in all ASD patients by dividing individuals into two groups of $\leq 10$ years old and $\geq 11$ years old, based on the onset of adolescence (71). 


\section{Differential methylation of retrotransposon subfamilies}

Methylation datasets were normalized using the single-sample normalized (ssNoob) method in minfi package (72). CpG sites within retrotransposons were identified using Illumina Infinium 450K annotation (23). To identify the variant-associated differential methylation of REs, probes with single nucleotide polymorphisms (SNPs) located at or within 10 base pairs of the target CpG site were included in the analysis. The CpG sites were mapped to LINE-1, Alu, half-L1 (HAL1), fossil Alu monomer (FAM), free right Alu monomer (FRAM), and free left Alu monomer (FLAM). Due to the evolution age of the REs, LINE-1 elements were clustered into oldest (L1M, mammalian-wide), intermediate (L1P, primate-specific), and youngest (L1HS, human-specific and L1PA, primate-amplified). Concomitantly, Alu elements were categorized into AluJ (oldest), AluS (intermediate) and AluY (youngest).

Mean $\beta$ value across all loci of REs was calculated as global DNAm in non-ASD, ASD with 16p11.2 del, and ASD with CHD8 variants. Differential methylation of LINE-1 and Alu subfamilies between 1) non-ASD vs ASD, 2) non-ASD vs ASD with $16 p 11.2$ del, and 3) non-ASD vs ASD with CHD8 variants, were identified. Differentially methylated positions (DMPs) to ASD were examined in the validation dataset. DMPs were identified in ASD, ASD with 16.p11.2 del, ASD with CHD8 variants and non-ASD with 16.p11.2 del, by two-tailed t-test with correction for false discovery rate (FDR) using the Benjamini-Hochberg $(\mathrm{BH})$ method (73) and significance defined as $\mathrm{P}_{\mathrm{FDR}} \leq 0.05$. To find the unique DMPs of each data set, the significant loci from 1) to 3) comparisons were computed to create Venn diagrams (https://bioinfogp.cnb.csic.es/tools/venny/).

\section{Differential gene expression analysis}

The expression data of ASD studies were obtained from the GEO DataSets. The data from each study were analyzed separately using the Multiple Experiment Viewer (MeV) program (microarray software suite) (74). Firstly, the data were filtered using a $70 \%$ cut-off filter to remove probes that were missing in $>30 \%$ of samples. The available data were then used for the identification of differentially expressed genes (DEGs) in ASD vs non-ASD cohort by using the Significance Analysis of Microarrays (SAM). The FDR and q-value less than $5 \%$ were considered as significantly DEGs.

RNA-sequencing (RNA-seq) data were obtained from the Sequence Read Archive database and re-analyzed using the Galaxy platform (https://usegalaxy.org/) (75). The quality control of RNA-seq data was assessed by fastp tool (76). The cleaned reads were then mapped to the human reference genome (GRCh38/hg38) using HISAT2 (77) and quantified using the Subread package FeatureCounts (78). Differential expression analysis was performed using the DESeq2 package (79). The read counts were normalized using the median ratio method of the DESeq2 and the remove unwanted variation (RUV) tool (77). The genes with a $p$-value $(p)$ with Benjamini-Hochberg correction of less than 0.05 were considered significant.

\section{Gene functions and pathway analysis}

To predict biological functions and gene regulatory networks associated with LINE-1 and Alu elements, a list of genes located nearby DMPs of LINE-1 and Alu elements for each ASD variant were submitted to the Ingenuity Pathway Analysis software (IPA: QIAGEN Inc.,https://www.qiagenbioinformatics.com/products/ingenuitypathway-analysis) (80). Gene regulatory networks were highlighted with $\log _{2}$ fold change of DNAm level.

\section{Identification of target loci in ASD with each genetic variant}

The target RE loci of ASD with each genetic variant were identified by taking the unique DMPs from the Venn diagrams and re-analyzing the different methylation of RE loci in ASD vs ASD with 16.p11.2 del or with CHD8 variants. We only selected loci which were significant in all three conditions (non-ASD vs ASD, ASD vs ASD with 16.p11.2 del and ASD vs ASD with CHD8 variants) by two-tailed t-test with correction for multiple hypothesis testing using the $\mathrm{BH}$ method and significance defined as 
$\mathrm{P}_{\mathrm{FDR}} \leq$ 0.05. Moreover, the DMRs, located nearby the significantly distinct DMPs, were identified in ASD with 16.p11.2 del or with $C H D 8$ variants.

\section{Statistical analyses}

Differentially methylated loci were identified by two-tailed t-tests with significance defined as $\mathrm{P}_{\mathrm{FDR}} \leq 0.05$ by the $\mathrm{BH}$ method or $p \leq 0.05$ for validation data due to low power in the analysis. Two-tailed t-tests were used to identify significant changes in age acceleration residuals among non-ASD and ASD. Pearson's correlation coefficient was used to compare DNAm Age and chronological age. Fisher's exact test was used to identify enrichment by genomic location of REs. DEGs were identified using SAM analysis with significance defined as FDR $\leq 0.05$ by the $\mathrm{BH}$ method. Gene function and pathway analysis were performed in IPA using Fisher's exact test with $\mathrm{BH}$ correction for multiple testing $\left(\mathrm{P}_{\mathrm{FDR}} \leq 0.05\right.$ was considered to be significant). All statistical analyses were performed in R (version 4.0.5) and RStudio (version 1.4.1103) using the ggplot2, plotROC, pheatmap, and GraphPad Prism (version 7.0b); data are presented as mean \pm SD, and $p \leq 0.05$ were considered to be significant.

\section{Results}

\section{Epigenetic age acceleration (EAA) changes in ASD blood}

DNAm Age was predicted using Horvath's method, which employs $353 \mathrm{CpG}$ sites (28). EAA was then estimated as the residual resulting from a multivariate model regressing the DNAm Age estimate on chronological age. Firstly, we correlated chronological age and DNAm Age in non-ASD ( $n=59$, combining non-ASD and non-ASD with 16.p11.2 del) and ASD ( $n=72$, combining ASD, ASD with 16p11.2 del, and ASD with CHD8 variants). The results show that DNAm Age was significantly correlated with chronological age in non-ASD $(r=0.72, p<0.0001)$, but the correlation was lower in the ASD cohort ( $r=0.49$, $p<0.0001$ ) (Figure 1A and 1B). The expected correlation found in the non-ASD group indicated that DNAm Age in our analyses can be determined EAA. The chronological age difference between the two cohorts was not significant (ASD: 9.94, non-ASD: 9.68, $p=0.723$ ) (Figure 1C), while the DNAm Age of the ASD cohort was considerably decreased (ASD: -0.84, nonASD: $0.84, p=0.00078$ ) (Figure 1D). Next, we classified non-ASD and ASD cohorts by $\leq 10$ years old and $\geq 11$ years old. As for previous comparisons, chronological age did not differ between ASD and non-ASD in both groups (Figure 1E). In the ASD cohort older than 11 years old, EAA shows a significant deceleration compared with an age-matched non-ASD cohort but this deceleration was not found in ASD younger than 11 years old (Figure 1F). These findings suggest that people with ASD were epigenetically younger than their chronological age which may be occurring in response to various environmental risk factors.

\section{DNAm profile of LINE-1 and Alu elements in the heterogeneous ASD}

A total of 22,352 probes mapping to LINE-1 and Alu elements were identified on the Infinium 450K platform for differential DNAm analysis. The analyses were performed for heterogeneous ASD, ASD with 16p11.2del, and ASD with CHD8 variants, versus non-ASD. Firstly, we measured the global methylation by combining all positions mapping to LINE-1 and Alu elements as the total of CpGs. In the comparison of heterogeneous ASD against non-ASD (Figure 2A), there was no significant difference in global methylation between these cohorts $(\Delta \beta=0.003, p=0.098)$. However, when we performed the methylation profile of REs by which LINE-1 and Alu positions were analyzed separately, we found that 2,802 (LINE-1) and 4,363 (A/u) DMPs were significantly differentially methylated $\left(P_{F D R}<0.05\right)$ in the heterogeneous ASD compared to non-ASD (Figure 2B and 2C, Figure 3). All these loci included 2,471 hypomethylated loci (LINE-1: 1,437 loci, Alu: 1,304 loci; $\mathrm{P}_{\mathrm{FDR}}<0.05$ ) and 4,424 hypermethylated loci (LINE-1:1,365 loci, Alu: 3,059 loci; $\left.\mathrm{P}_{\mathrm{FDR}}<0.05\right)$. Due to the different activity of subfamilies of RE, LINE-1 and Alu elements were clustered by evolution age into three categories including old age (L1M, AluJ), intermediate age (L1P, L1PB, AluS), young age (L1HS, L1PA, AluY), and related (HAL1, FAM, FLAM, FRAM). The methylation of LINE-1 and Alu 
elements were changed in a subfamily-specific manner. We discovered that LINE-1 was considerably hypermethylated in young and intermediate age families, including $\operatorname{L} 1 \mathrm{H}(\Delta \beta=0.013, p=0.00001)$ and $\operatorname{L} 1 \mathrm{P}(\Delta \beta=0.005, p=0.027)$, but HAL1 was hypomethylated $(\Delta \beta=-0.003, p=0.03)$ (Figure $2 D)$. Methylation of Alu elements was significantly hypermethylated in the old and intermediate age families: $A / u J(\Delta \beta=0.006, p=0.004)$ and $A / u S(\beta=0.005, p=0.016)$, respectively (Figure 2E). These findings indicated that methylation of LINE-1 and Alu elements in the heterogeneous ASD was altered in family-and locus- specific manner rather than globally.

\section{LINE-1 and Alu methylation signatures in the homogeneous ASD (16p11.2del and CHD8 variants)}

Due to the heterogeneity in the ASD population, we also intended to investigate the methylation profile of LINE-1 and Alu elements in genetically homogeneous ASD, as identified in the original article of GSE113967, including ASD individuals with 16p11.2del $(n=7)$ and CHD8 variant $(n=15)$. As for the results of ASD with 16p11.2del compared with non-ASD, we found no significant changes in the global methylation compared with non-ASD $(\Delta \beta=-0.002, p=0.771$, Figure 4A). However, the analyses identified 70 significantly locus-specific DMPs of REs in ASD with 16p11.2del including 27 DMPs at LINE-1 (5 hypomethylated loci, 22 hypermethylated loci, Figure 4B) and 43 DMPs at Alu elements (23 hypomethylated loci, 20 hypermethylated loci, Figure 4C). When LINE-1 and Alu positions were categorized into families, there was no significant difference in methylation of LINE-1 and Alu elements by family (Figure 4D and 4E).

Subsequently, we analyzed data for ASD with $C H D 8$ variants by using the same approach. We found that global methylation was exclusively hypomethylated in the ASD with $C H D 8$ variants $(\Delta \beta=-0.006, p=0.042$, Figure $5 A)$. Analyzing by the position, the majority of DMPs at LINE-1 and Alu elements were hypomethylated (616 loci or $\left.88.63 \%, \mathrm{P}_{\mathrm{FDR}}<0.05\right)$ of the total identified 695 DMPs observed (Figure 5B and 5C). Among all significant DMPs in the ASD with CHD8 variants, 528 DMPs were mapped to LINE-1, while 167 DMPs were Alu elements. Moreover, changes in LINE-1 and Alu methylation regarding to their families were observed in ASD with CHD8 variants. In contrast to the differences found in the heterogeneous ASD, young and intermediate age LINE-1 families were significantly hypomethylated including $\operatorname{L} 1 \mathrm{H}(\Delta \beta=-0.015, p=0.0038)$ and $\mathrm{L} 1 \mathrm{P}(\Delta \beta=$ $-0.010, p=0.0186$ ) (Figure 5D). Hypomethylation of Alu elements was also observed in old age, intermediated age, and related families: AluJ $(\Delta \beta=-0.010, p=0.0154), \operatorname{AluS}(\Delta \beta=-0.008, p=0.0443), \operatorname{FAM}(\Delta \beta=-0.008, p=0.0399), \operatorname{FRAM}(\Delta \beta=$ $-0.007, p=0.02551$ ) respectively (Figure $5 \mathrm{E}$ ). These findings suggest that DNAm signatures were a widespread reduction in LINE-1 and Alu regions which occurred at a specific family in the ASD with CHD8 variants but not in ASD with 16p11.2del.

\section{Genomic distribution of LINE-1 and Alu methylation in heterogenous and homogenous ASD.}

To determine the differential DNAm of LINE-1 and Alu elements by genomic features, we performed enrichment analysis using Fisher's exact test. CpG positions at LINE-1 and Alu elements were categorized to 1500 and 200 within the transcriptional start site (TSS1500 and TSS200, respectively), the $5^{\prime}$ untranslated region ( $5^{\prime}$ UTR), the first exon (1st exon), gene body (Body), and 3' untranslated region (3'UTR). In the heterogeneous ASD signatures, CpG sites at LINE-1 were significantly enriched in TSS1500 ( $p=0.0005)$ and Body $(p<0.0001)$ (see Additional file 2A). Whereas Alu elements were significantly enriched in TSS1500 ( $p<0.0001), 5^{\prime} \mathrm{UTR}(p<0.0001)$, Body $(p<0.0001)$, and 3'UTR $(p=0.0086)$ (see Additional file 3A). However, DNAm across all retrotransposons by genomic location did not significantly differ between non-ASD and heterogenous ASD (see Additional file 2B and 3B). DNAm signatures of the ASD with 16p11.2del and CHD8 variants were significantly enriched in Body $(p=0.04)$ and TSS1500 $(p<0.0001)$ respectively (see Additional file 4: Figure S5B, S5C). This

result shows that the changes of probes mapping to TSS1500 and gene bodies are more likely to have a functional impact on gene expression in ASD in both heterogenous and homogenous ASD. 


\section{Biological functions and pathways of LINE-1 and Alu methylation signatures in ASD and ASD variants.}

To determine the biological significance of LINE-1 and Alu methylation signatures identified in each ASD cohort, we predicted the biological function and pathway of genes located nearby DMPs of LINE-1 and Alu elements using IPA software. We found that neurological diseases were significantly enriched among genes associated with LINE-1 and Alu methylation signatures in the heterogeneous ASD ( $p$ range: 0.00495 - 3.33E-26, 2274 genes) and ASD with CHD8 variants ( $p$ range: $0.0258-0.000117$, 302 genes) as shown in Additional file 5 and 6 . The categories ASD and intellectual disability were exclusively associated with LINE-1 and Alu methylation signatures in the heterogeneous ASD ( $p=2.56 \mathrm{E}-06,253$ genes). Whereas Huntington's disease, familial encephalopathy, and brain lesion were commonly associated with both ASD signatures. For ASD with 16p11.2del variant, LINE-1 and Alu methylation signatures in this cohort were significantly associated with developmental disorders ( $p$ range: $0.0393-0.00222,9$ genes) (see Additional file 7 ). However, only one gene was associated with the disease, possibly caused by a small number of genes associated with LINE-1 and Alu methylation of this ASD variant. Additionally, we discovered that several canonical pathways linked to ASD were associated with genes located nearby LINE-1 and Alu methylation signatures in each ASD cohort. More precisely, we found that the a-adrenergic signaling pathway was significantly associated in the heterogeneous ASD $(p=0.00269,28$ genes $)$ and ASD with CHD 8 variants $(p=0.00646,7$ genes). Axonal guidance signaling pathway involved in nervous system development was significantly associated with LINE1 and Alu methylation signatures of ASD with 16p11.2del and $C H D 8$ variants. These results indicate that genes associated with LINE-1 and Alu methylation signatures in ASD were involved with neurological diseases and ASD-comorbid disorders as well as canonical pathways known to be implicated in ASD. The list of all significant biological functions and pathways in each ASD variant is shown in Additional file 5-7.

Interactome networks or gene regulatory networks revealed the interaction of genes located nearby LINE-1 and Alu methylation signatures of each ASD variant. The functions and pathways implicated in ASD were highlighted in the networks. The interactome of the heterogeneous ASD was associated with ASD and mental retardation, as well as canonical pathways implicated in ASD such as retinoic acid receptor (RAR) and AMP-activated protein kinase (AMPK) signaling (Figure 6). In ASD with 16p11,2del, we found that the interactome related to axonal guidance and sirtuin signaling pathway (see Additional file 8: Figure S8A). The interactome of ASD with $C H D 8$ was related to familial encephalopathy and movement disorder which conditions found in ASD individuals $(29,30)$. The interactomes were also associated with neuronal function including axonal guidance and synaptogenesis signaling pathways (see Additional file 8: Figure S8B and S8C).

\section{Identification of unique target loci located nearby LINE-1 and $A / u$ signatures in heterogeneous ASD}

To investigate the functional impact of locus-specific LINE-1 and Alu methylation to target gene or neighboring gene expression in the ASD, we identified DEGs from multiple ASD studies obtained from the GEO DataSets. This approach reflected the heterogeneity of the ASD population because these studies were compiled from a different ASD cohort. There were 12,419 DEGs identified from seven datasets including four studies (one study used peripheral blood samples and three studies used post-mortem brain tissues from ASD individuals) (see Additional file 1). We subsequently overlapped the list of DEGs with differentially methylated genes (DMGs: genes located nearby LINE-1 and Alu signatures). The overlapping revealed 1,847 DMGs in the heterogeneous ASD that were differentially expressed in several ASD studies, with 155 of them being autism-related genes in the SFARI database. We identified 43 top DMGs, $|\Delta \beta| \geq 5 \%$, inversely related to gene expression, and differentially expressed in at least two studies (Table 2). Interestingly, two of the top DMGs, potassium voltage-gated channel subfamily Q member 3 (KCNQ3) and ubiquitin conjugating enzyme E2 H (UBE2H), were genes in the SFARI database and were enriched in the gene regulatory network related to ASD and mental retardation (Figure 6). 
The genomic regions of LINE-1 and Alu methylation signatures within the DMGs are shown in Figure 7. We identified DMRs by mapping all probes located nearby LINE-1 and Alu signatures using the UCSC genome browser. The findings revealed that AluSg7 (cg16926147), which is located on the gene body of the KCNQ3 gene (Figure 7A), was hypermethylated and KCNQ3 expression level was significantly reduced in blood and post-mortem brain tissues. Interestingly, we discovered that several probes in this region, including those in the promoter region were not changed. This result suggests that LINE-1 and Alu methylation at DMRs may facilitate gene expression indicated by the inverse relationship between LINE-1/Alu methylation and gene expression. As well as AluY (cg08998414) within UBE2H gene (Figure 7B) and L1PA3 (cg24094412) within hyperpolarization activated cyclic nucleotide gated potassium channel 1 (HCN1) (Figure 7C), we also observed that AluY and L1PA3 methylation were inversely related to the gene expression levels in both blood and brain tissues of ASD cohort. Moreover, we found several DMGs that were not reported in the SFARI database but the expression of these DMGs in the blood and post-mortem brain tissues was inversely related to LINE-1 and Alu methylation such as N-deacetylase and Nsulfotransferase 1 (NDST1) (cg12611243: L1MC1), ubiquitin specific peptidase 6 (USP6) (cg23416909: L1M5), and formin binding protein 1 (FNBP1) (cg13916261: AluSg). These results suggest that DMPs at LINE-1 and Alu elements may affect the expression of genes located nearby these DMPs in the heterogeneous ASD cohort.

Table 2 Utilized gene expression microarrays/RNA-sequencing for the differential gene expression analysis of the target genes of heterogeneous ASD. 
Methylome data

Transcriptome data

\begin{tabular}{|c|c|c|c|c|c|c|c|c|}
\hline Probe ID & Elements & Delta & $P_{F D R}$ & GSE & Gene ID & Gene & $\log 2 \mathrm{FC}$ & q-value \\
\hline cg02571470 & L1MC5 & 0.061 & $\begin{array}{l}1.61 \mathrm{E}- \\
05\end{array}$ & GSE18123 & 1563708_at & SFXN5 & -0.348 & 0.0164 \\
\hline cg02571470 & L1MC5 & 0.061 & $\begin{array}{l}1.61 \mathrm{E}- \\
05\end{array}$ & GSE18123 & 241999_at & SFXN5 & -0.407 & 0.0386 \\
\hline cg21314304 & AluJb & 0.054 & $\begin{array}{l}2.68 \mathrm{E}- \\
05\end{array}$ & GSE25507 & 226298_at & $R U N D C 1$ & -0.141 & 0.0272 \\
\hline cg21314304 & AluJb & 0.054 & $\begin{array}{l}2.68 \mathrm{E}- \\
05\end{array}$ & GSE28521_FC & ILMN_1733875 & $R U N D C 1$ & -0.234 & 0.0319 \\
\hline cg21314304 & AluJb & 0.054 & $\begin{array}{l}2.68 \mathrm{E}- \\
05\end{array}$ & GSE59288 & 146923 & RUNDC1 & -0.452 & 0.0001 \\
\hline cg04668642 & AluSx & 0.051 & $\begin{array}{l}2.68 \mathrm{E}- \\
05\end{array}$ & GSE64018 & ENSG00000166780 & C16orf45 & -0.224 & 0.0421 \\
\hline cg04668642 & AluSx & 0.051 & $\begin{array}{l}2.68 \mathrm{E}- \\
05\end{array}$ & GSE28521_FC & ILMN_1687821 & C16orf45 & -0.256 & 0.0287 \\
\hline cg02616069 & $A l u J b$ & 0.069 & $\begin{array}{l}3.02 \mathrm{E}- \\
05\end{array}$ & GSE18123 & 215584_at & HECW1 & -0.259 & 0.0068 \\
\hline cg02616069 & $A l u J b$ & 0.069 & $\begin{array}{l}3.02 \mathrm{E}- \\
05\end{array}$ & GSE59288 & 23072 & HECW1 & -0.805 & $<0.0001$ \\
\hline cg15531814 & AluJo & 0.059 & $\begin{array}{l}4.42 \mathrm{E}- \\
05\end{array}$ & GSE89594 & A_23_P310257 & $K L K 2$ & -0.161 & 0.0176 \\
\hline cg15531814 & AluJo & 0.059 & $\begin{array}{l}4.42 \mathrm{E}- \\
05\end{array}$ & GSE18123 & 1555545_at & $K L K 2$ & -0.138 & 0.0254 \\
\hline $\operatorname{cg} 11204311$ & AluJr & 0.060 & $\begin{array}{l}4.54 \mathrm{E}- \\
05\end{array}$ & GSE28521_FC & ILMN_1679796 & TOMM20 & -0.506 & 0.0110 \\
\hline cg11204311 & AluJr & 0.060 & $\begin{array}{l}4.54 \mathrm{E}- \\
05\end{array}$ & GSE59288 & 9804 & TOMM20 & -0.176 & 0.0438 \\
\hline cg11204311 & AluJr & 0.060 & $\begin{array}{l}4.54 \mathrm{E}- \\
05\end{array}$ & GSE64018 & ENSG00000173726 & TOMM20 & -0.231 & 0.0459 \\
\hline cg04027778 & AluSc & 0.054 & $\begin{array}{l}4.66 \mathrm{E}- \\
05\end{array}$ & GSE28521_TC & ILMN_1712705 & $R A B 40 C$ & -0.258 & 0.0313 \\
\hline cg04027778 & AluSc & 0.054 & $\begin{array}{l}4.66 \mathrm{E}- \\
05\end{array}$ & GSE18123 & 227269_s_at & $R A B 40 C$ & -0.429 & 0.0133 \\
\hline cg04027778 & AluSc & 0.054 & $\begin{array}{l}4.66 \mathrm{E}- \\
05\end{array}$ & GSE18123 & 1569396_at & $R A B 40 C$ & -0.309 & 0.0025 \\
\hline cg04027778 & AluSc & 0.054 & $\begin{array}{l}4.66 \mathrm{E}- \\
05\end{array}$ & GSE59288 & 57799 & $R A B 40 C$ & -0.322 & 0.0131 \\
\hline cg06719602 & AluSx 1 & 0.050 & $\begin{array}{l}5.57 \mathrm{E}- \\
05\end{array}$ & GSE28521_FC & ILMN_1706238 & CSE1L & -0.202 & 0.0384 \\
\hline cg06719602 & AluSx 1 & 0.050 & $\begin{array}{l}5.57 \mathrm{E}- \\
05\end{array}$ & GSE28521_FC & ILMN_1665797 & CSE1L & -0.184 & 0.0449 \\
\hline cg23935361 & AluJb & 0.066 & $\begin{array}{l}5.82 \mathrm{E}- \\
05\end{array}$ & GSE42133 & ILMN_1738093 & RNFT2 & -0.102 & 0.0090 \\
\hline cg23935361 & $A l u J b$ & 0.066 & $\begin{array}{l}5.82 \mathrm{E}- \\
05\end{array}$ & GSE59288 & 84900 & RNFT2 & -0.259 & 0.0467 \\
\hline
\end{tabular}




\begin{tabular}{|c|c|c|c|c|c|c|c|c|}
\hline $\operatorname{cg} 17429234$ & AluSp & 0.061 & $\begin{array}{l}6.88 \mathrm{E}- \\
05\end{array}$ & GSE59288 & 1780 & DYNC1/1 & -0.852 & $<0.0001$ \\
\hline cg17429234 & AluSp & 0.061 & $\begin{array}{l}6.88 \mathrm{E}- \\
05\end{array}$ & GSE28521_FC & ILMN_1690397 & DYNC111 & -0.516 & 0.0081 \\
\hline cg17429234 & AluSp & 0.061 & $\begin{array}{l}6.88 \mathrm{E}- \\
05\end{array}$ & GSE64018 & ENSG00000158560 & DYNC1/1 & -0.324 & 0.0410 \\
\hline cg23376467 & AluSq & 0.077 & $\begin{array}{l}7.13 \mathrm{E}- \\
05\end{array}$ & GSE18123 & 1556907_at & ZNF474 & -0.292 & 0.0346 \\
\hline cg23376467 & AluSq & 0.077 & $\begin{array}{l}7.13 \mathrm{E}- \\
05\end{array}$ & GSE89594 & A_33_P3360565 & ZNF474 & -0.205 & 0.0200 \\
\hline cg06471678 & AluYc & -0.065 & $\begin{array}{l}7.85 \mathrm{E}- \\
05\end{array}$ & GSE25507 & 233694_at & HSPA1L & 0.111 & 0.0307 \\
\hline cg06471678 & AluYc & -0.065 & $\begin{array}{l}7.85 \mathrm{E}- \\
05\end{array}$ & GSE42133 & ILMN_1654566 & HSPA1L & 0.107 & 0.0300 \\
\hline cg15074424 & AluS $x 1$ & 0.083 & $\begin{array}{l}1.01 \mathrm{E}- \\
04\end{array}$ & GSE28521_FC & ILMN_2212354 & WDR46 & -0.201 & 0.0364 \\
\hline cg15074424 & AluS $x 1$ & 0.083 & $\begin{array}{l}1.01 \mathrm{E}- \\
04\end{array}$ & GSE28521_TC & ILMN_2212354 & WDR46 & -0.303 & 0.0335 \\
\hline cg02747612 & AluSq & -0.068 & $\begin{array}{l}1.02 \mathrm{E}- \\
04\end{array}$ & GSE42133 & ILMN_1802053 & ZNF91 & 0.162 & 0.0158 \\
\hline cg02747612 & AluSq & -0.068 & $\begin{array}{l}1.02 \mathrm{E}- \\
04\end{array}$ & GSE18123 & 236128_at & ZNF91 & 0.306 & 0.0474 \\
\hline $\operatorname{cg} 13916261$ & AluSg & -0.100 & $\begin{array}{l}1.05 \mathrm{E}- \\
04\end{array}$ & GSE59288 & 23048 & FNBP1 & 0.354 & 0.0128 \\
\hline cg13916261 & Alusg & -0.100 & $\begin{array}{l}1.05 \mathrm{E}- \\
04\end{array}$ & GSE42133 & ILMN_1797342 & FNBP1 & 0.156 & $<0.0001$ \\
\hline cg02827046 & L1PA16 & 0.099 & $\begin{array}{l}1.17 \mathrm{E}- \\
04\end{array}$ & GSE89594 & A_33_P3420900 & PATE2 & -0.221 & 0.0142 \\
\hline cg02827046 & L1PA16 & 0.099 & $\begin{array}{l}1.17 \mathrm{E}- \\
04\end{array}$ & GSE42133 & ILMN_2133784 & PATE2 & -0.157 & 0.0236 \\
\hline cg07930329 & AluS $\times 3$ & 0.056 & $\begin{array}{l}1.21 \mathrm{E}- \\
04\end{array}$ & GSE59288 & 10055 & SAE1 & -0.295 & 0.0064 \\
\hline cg07930329 & AluS $\times 3$ & 0.056 & $\begin{array}{l}1.21 \mathrm{E}- \\
04\end{array}$ & GSE28521_TC & ILMN_1657204 & SAE1 & -0.194 & 0.0417 \\
\hline cg07930329 & AluSx3 & 0.056 & $\begin{array}{l}1.21 \mathrm{E}- \\
04\end{array}$ & GSE64018 & ENSG00000142230 & SAE1 & -0.194 & 0.0395 \\
\hline cg16926147 & AluSg7 & 0.062 & $\begin{array}{l}1.31 \mathrm{E}- \\
04\end{array}$ & GSE18123 & 206573_at & KCNQ3 & -0.093 & 0.0182 \\
\hline cg16926147 & AluSg7 & 0.062 & $\begin{array}{l}1.31 \mathrm{E}- \\
04\end{array}$ & GSE59288 & 3786 & KCNQ3 & -0.634 & 0.0003 \\
\hline cg08499057 & AluJo & 0.057 & $\begin{array}{l}1.63 \mathrm{E}- \\
04\end{array}$ & GSE28521_FC & ILMN_1781999 & $A B C F 2$ & -0.164 & 0.0426 \\
\hline cg08499057 & AluJo & 0.057 & $\begin{array}{l}1.63 \mathrm{E}- \\
04\end{array}$ & GSE28521_FC & ILMN_1669201 & $A B C F 2$ & -0.168 & 0.0426 \\
\hline cg08499057 & AluJo & 0.057 & $\begin{array}{l}1.63 \mathrm{E}- \\
04\end{array}$ & GSE28521_TC & ILMN_1669201 & $A B C F 2$ & -0.202 & 0.0372 \\
\hline cg08499057 & AluJo & 0.057 & $1.63 \mathrm{E}-$ & GSE18123 & 207623_at & $A B C F 2$ & -0.321 & 0.0053 \\
\hline
\end{tabular}


04

\begin{tabular}{lllllllll}
\hline cg08998414 & AluY & -0.106 & $\begin{array}{l}1.85 \mathrm{E}- \\
04\end{array}$ & GSE64018 & ENSG00000186591 & UBE2H & 0.130 & 0.0352 \\
\hline cg08998414 & AluY & -0.106 & $\begin{array}{l}1.85 \mathrm{E}- \\
04\end{array}$ & GSE42133 & ILMN_1757644 & UBE2H & 0.126 & 0.0258 \\
\hline cg08998414 & AluY & -0.106 & $\begin{array}{l}1.85 \mathrm{E}- \\
04\end{array}$ & GSE25507 & 222419_x_at & UBE2H & 0.171 & 0.0230
\end{tabular}

\section{Identification of unique target loci located nearby LINE-1 and Alu signatures in ASD variants.}

To investigate the effects of unique LINE-1 and Alu methylation signatures to target gene or neighboring gene expression in the genetically homogeneous ASD, we obtained 39 and 101 DMPs that were found exclusively in the ASD with 16.p11.2 del and $C H D 8$ variants, respectively (see Additional file 4: Figure S4A). We re-analyzed them for ASD variant versus the heterogenous ASD. Next, we conducted the same strategy used for the heterogeneous ASD to select the candidate DMPs by overlapping with the transcriptome data. The overlapping of unique DMPs with transcriptome data revealed 11 and 31 unique DMGs in the ASD with 16.p11.2 del and CHD8 variants, respectively (Table 3 and 4). Among the unique DMGs, we found several genes linked to neurodevelopmental disorder and ASD, including XK related 6 (XKR6) (Figure 8A), zinc finger protein 107 (ZNF107) (Figure 8B), and myeloma-overexpressed gene 2 protein (MYEOV2) (Figure 8C) in the ASD with 16.p11.2 del. The significant DMPs at AluY (cg21300361) within XKR6 was hypermethylated, while as AluSq (cg01772945) within ZNF107 and L1MB3 (cg13749477) within MYEOV2 were hypomethylated. Interestingly, these genes were differentially expressed in the blood transcriptome of multiple ASD cohorts, and their expression was inversely relative to LINE-1 and Alu methylation levels.

For ASD with CHD8 variants, we found that all LINE-1 and Alu elements located on candidate genes were markedly hypomethylated, as expected from global and family-specific methylation levels. These DMPs consist of L1MC5 (cg22706070) within Euchromatic Histone Lysine Methyltransferase 2 (EHMT2) (Figure 9A), AluJo (cg06421197) within caspase 1 (CASP1) (Figure 9B), and AluSx (cg18699242, cg01963623, cg02169692) within ubiquitin-specific peptidase 18 (USP18) (Figure 9C). EHMT2 was significantly increased in the blood of ASD, while CASP1 was increased in both the blood and brain of multiple ASD cohorts (one probe was decreased). These changes were inversely relative to LINE-1 and Alu methylation levels within that gene. We found that the expression of USP18 was not inversely relative to AluSX methylation located on the gene. Additionally, the DMRs of XKR6, ZNF107, MYEOV2, EHMT2, and CASP1 genes revealed LINE-1 and Alu probes as well as non-LINE-1/Alu probes located in the same DMRs (Figure 8A-C and Figure 9A-B).

Table 3 Utilized gene expression microarrays/RNA-sequencing for the differential gene expression analysis of the target genes of ASD with 16p11.2 deletion. 


\begin{tabular}{lllllllll}
\multicolumn{1}{l}{ Methylome data } & \multicolumn{7}{c}{ Transcriptome data } \\
\hline Probe ID & Elements & Delta & P FDR & GSE & Gene ID & Gene & log2FC & q-value \\
\hline cg22062537 & L1MB3 & -0.079 & 0.0002 & GSE25507 & 1553515_at & MYEOV2 & 0.107 & 0.0496 \\
\hline cg13749477 & L1MB3 & -0.038 & 0.0004 & GSE25507 & 1553515_at & MYEOV2 & 0.107 & 0.0496 \\
\hline cg07628769 & L1MB3 & -0.101 & 0.0028 & GSE25507 & 1553515_at & MYEOV2 & 0.107 & 0.0496 \\
\hline cg01772945 & AluSq & -0.048 & 0.0003 & GSE18123 & 205739_x_at & ZNF107 & 0.382 & 0.0017 \\
\hline cg09168728 & HAL1 & 0.042 & 0.0039 & GSE18123 & 202651_at & LPGAT1 & 0.160 & 0.0038 \\
\hline cg21300361 & AluY & 0.024 & 0.0139 & GSE18123 & 1553640_at & XKR6 & -0.949 & 0.0206 \\
\hline cg21300361 & AluY & 0.024 & 0.0139 & GSE25507 & 1553640_at & XKR6 & 0.134 & 0.0112 \\
\hline cg01270736 & AluJb & -0.037 & 0.0167 & GSE25507 & 207289_at & MMP25 & 0.193 & $<0.0001$ \\
\hline cg26620682 & L1PA2 & -0.058 & 0.0206 & GSE28521_FC & ILMN_1765641 & SEMA3A & -0.200 & 0.0343 \\
\hline cg26620682 & L1PA2 & -0.058 & 0.0206 & GSE59288 & 10371 & SEMA3A & -0.590 & 0.0139 \\
\hline cg05073382 & L1MA7 & 0.153 & 0.0290 & GSE89594 & A_23_P258912 & MYOM2 & -1.611 & 0.0114 \\
\hline cg11438448 & AluSX4 & -0.014 & 0.0364 & GSE18123 & 233429_at & SPEF2 & -0.285 & 0.0484 \\
\hline cg10059324 & L1MC4a & 0.050 & 0.0368 & GSE59288 & 8863 & PER3 & 0.296 & 0.0269 \\
\hline cg11859345 & AluSp & -0.031 & 0.0474 & GSE18123 & 228766_at & CD36 & 0.171 & 0.0249 \\
\hline cg11859345 & AluSp & -0.031 & 0.0474 & GSE18123 & 206488_s_at & CD36 & 0.273 & 0.0076 \\
\hline cg11859345 & AluSp & -0.031 & 0.0474 & GSE25507 & 209554_at & CD36 & 0.099 & 0.0131 \\
\hline cg25283432 & L1MA8 & 0.004 & 0.0482 & GSE25507 & 232421_at & SCARB1 & 0.116 & 0.0189
\end{tabular}

Table 4 Utilized gene expression microarrays/RNA-sequencing for the differential gene expression analysis of the target genes of ASD with CHD8 variants. 
Methylome data

Transcriptome data

\begin{tabular}{|c|c|c|c|c|c|c|c|c|}
\hline Probe ID & Elements & Delta & $P_{F D R}$ & GSE & Gene ID & Gene & $\log 2 \mathrm{FC}$ & q-value \\
\hline cg10071848 & AluJb & -0.033 & 0.0439 & GSE59288 & 5826 & $A B C D 4$ & 0.39 & 0.0078 \\
\hline cg07730946 & $A l u J b$ & -0.027 & 0.0128 & GSE42133 & ILMN_1684585 & ACSL 1 & -0.24 & 0.0344 \\
\hline $\operatorname{cg} 12934569$ & AluSx & -0.043 & 0.0319 & GSE42133 & ILMN_1684585 & ACSL 1 & -0.24 & 0.0344 \\
\hline cg11111835 & L1MA3 & -0.020 & 0.0359 & GSE18123 & 1553603_s_at & ATL2 & 0.28 & 0.0248 \\
\hline cg11111835 & L1MA3 & -0.020 & 0.0359 & GSE18123 & 237968_at & ATL2 & -0.25 & 0.0492 \\
\hline cg04154142 & AluSx 1 & -0.063 & 0.0110 & GSE42133 & ILMN_1672596 & $B C A R 1$ & -0.09 & 0.0127 \\
\hline cg04154142 & AluSx 1 & -0.063 & 0.0110 & GSE18123 & 223116_at & $B C A R 1$ & -0.26 & 0.0226 \\
\hline $\operatorname{cg} 14115346$ & L1MB3 & -0.038 & 0.0201 & GSE18123 & 205750_at & $B P H L$ & 0.28 & 0.0097 \\
\hline cg10211626 & AluSx 1 & -0.059 & 0.0028 & GSE59288 & 79640 & C22orf46 & 0.56 & 0.0002 \\
\hline $\operatorname{cg} 17129519$ & AluSg & 0.097 & 0.0360 & GSE64018 & ENSG00000204564 & C6orf136 & -0.18 & 0.0430 \\
\hline cg17129519 & AluSg & 0.097 & 0.0360 & GSE28521_TC & ILMN_1813236 & C6orf136 & -0.30 & $<0.0001$ \\
\hline cg06421197 & AluJo & -0.034 & 0.0266 & GSE18123 & 206011_at & CASP1 & 0.14 & 0.0122 \\
\hline cg06421197 & AluJo & -0.034 & 0.0266 & GSE42133 & ILMN_2326509 & CASP1 & -0.16 & 0.0258 \\
\hline cg06421197 & AluJo & -0.034 & 0.0266 & GSE42133 & ILMN_2326512 & CASP1 & -0.17 & 0.0213 \\
\hline cg06421197 & AluJo & -0.034 & 0.0266 & GSE42133 & ILMN_1727762 & CASP1 & -0.25 & $<0.0001$ \\
\hline cg06421197 & AluJo & -0.034 & 0.0266 & GSE59288 & 834 & CASP1 & 0.61 & 0.0096 \\
\hline cg13606720 & L1ME3F & -0.044 & 0.0125 & GSE42133 & ILMN_3248676 & CBWD3 & -0.11 & 0.0180 \\
\hline cg11379605 & $A l u J b$ & -0.033 & 0.0359 & GSE59288 & 965 & CD58 & 0.47 & 0.0159 \\
\hline cg11379605 & $A l u J b$ & -0.033 & 0.0359 & GSE18123 & 222061_at & $C D 58$ & 0.36 & 0.0256 \\
\hline cg11379605 & $A / u J b$ & -0.033 & 0.0359 & GSE64018 & ENSG00000116815 & $C D 58$ & 0.50 & 0.0328 \\
\hline cg09425611 & L1MB7 & -0.028 & 0.0360 & GSE18123 & 209616_s_at & CES1 & 0.44 & 0.0025 \\
\hline cg00053536 & AluSx & -0.028 & 0.0462 & GSE25507 & 206274_s_at & CROCC & 0.12 & 0.0458 \\
\hline cg09604414 & AluSx & -0.067 & 0.0336 & GSE25507 & 229079_at & EHMT2 & 0.09 & 0.0471 \\
\hline cg22706070 & L1MC5 & -0.008 & 0.0496 & GSE25507 & 229079_at & EHMT2 & 0.09 & 0.0471 \\
\hline cg14570121 & FLAM_A & -0.031 & 0.0375 & GSE59288 & 2068 & $E R C C 2$ & -0.26 & 0.0149 \\
\hline cg24803614 & AluSc8 & 0.051 & 0.0341 & GSE59288 & 2495 & FTH1 & 0.42 & 0.0045 \\
\hline cg03085932 & L1PA6 & -0.043 & 0.0459 & GSE25507 & 220249_at & HYAL4 & 0.12 & 0.0179 \\
\hline cg08005007 & L1HS & -0.031 & 0.0095 & GSE18123 & 235111_at & LSAMP & -0.23 & 0.0386 \\
\hline cg08005007 & L1HS & -0.031 & 0.0095 & GSE18123 & 229244_at & LSAMP & -0.25 & 0.0303 \\
\hline cg22993878 & L1MB3 & -0.021 & 0.0080 & GSE25507 & 1553515_at & MYEOV2 & 0.11 & 0.0496 \\
\hline cg03514928 & L1PA17 & -0.022 & 0.0470 & GSE59288 & 4897 & NRCAM & -0.31 & 0.0104 \\
\hline cg20723844 & AluY & -0.061 & 0.0163 & GSE42133 & ILMN_1789616 & NUPL2 & -0.09 & 0.0221 \\
\hline cg00984715 & AluSq & -0.049 & 0.0224 & GSE59288 & 5465 & PPARA & 0.51 & 0.0149 \\
\hline
\end{tabular}




\begin{tabular}{|c|c|c|c|c|c|c|c|c|}
\hline cg00984715 & AluSq & -0.049 & 0.0224 & GSE18123 & 1560981_a_at & PPARA & -0.24 & 0.0256 \\
\hline cg00984715 & AluSq & -0.049 & 0.0224 & GSE18123 & 1558631_at & PPARA & -0.46 & 0.0091 \\
\hline cg24351819 & AluSp & -0.026 & 0.0474 & GSE59288 & 55170 & PRMT6 & -0.33 & 0.0057 \\
\hline cg09521141 & AluSg7 & -0.045 & 0.0263 & GSE28521_FC & ILMN_1765641 & SEMAЗA & -0.20 & 0.0343 \\
\hline cg09521141 & AluSg7 & -0.045 & 0.0263 & GSE59288 & 10371 & SEMA3A & -0.59 & 0.0139 \\
\hline cg26620682 & L1PA2 & -0.041 & 0.0346 & GSE28521_FC & ILMN_1765641 & SEMA3A & -0.20 & 0.0343 \\
\hline cg26620682 & L1PA2 & -0.041 & 0.0346 & GSE59288 & 10371 & SEMA3A & -0.59 & 0.0139 \\
\hline cg18221988 & L1PA2 & -0.043 & 0.0499 & GSE18123 & 210804_x_at & $S L C 8 A 1$ & -0.30 & 0.0392 \\
\hline cg18221988 & L1PA2 & -0.043 & 0.0499 & GSE25507 & 1565306_a_at & SLC8A1 & 0.18 & 0.0189 \\
\hline cg18221988 & L1PA2 & -0.043 & 0.0499 & GSE59288 & 6546 & SLC8A1 & -0.41 & 0.0245 \\
\hline cg18221988 & L1PA2 & -0.043 & 0.0499 & GSE18123 & 235518_at & $S L C 8 A 1$ & 0.29 & 0.0057 \\
\hline cg18221988 & L1PA2 & -0.043 & 0.0499 & GSE64018 & ENSG00000183023 & $S L C 8 A 1$ & -0.26 & 0.0430 \\
\hline cg07020453 & L1MDa & -0.009 & 0.0457 & GSE18123 & 217968_at & TSSC1 & -0.21 & 0.0185 \\
\hline cg08428949 & AluSz & -0.028 & 0.0124 & GSE64018 & ENSG00000128881 & TTBK2 & -0.15 & 0.0352 \\
\hline cg01810763 & AluSx 1 & -0.053 & 0.0442 & GSE64018 & ENSG00000198431 & TXNRD1 & 0.30 & 0.0443 \\
\hline cg11191744 & AluY & -0.050 & 0.0457 & GSE64018 & ENSG00000198431 & TXNRD1 & 0.30 & 0.0443 \\
\hline cg02169692 & AluSx & -0.109 & 0.0275 & GSE42133 & ILMN_3240420 & USP18 & -0.31 & 0.0250 \\
\hline cg02169692 & AluSx & -0.109 & 0.0275 & GSE42133 & ILMN_3240420 & USP18 & -0.31 & 0.0250 \\
\hline cg18699242 & AluSx & -0.106 & 0.0448 & GSE42133 & ILMN_3240420 & USP18 & -0.31 & 0.0250 \\
\hline cg18699242 & AluSx & -0.106 & 0.0448 & GSE42133 & ILMN_3240420 & USP18 & -0.31 & 0.0250 \\
\hline cg15726387 & AluSz6 & -0.044 & 0.0950 & GSE18123 & 220079_s_at & USP48 & 0.10 & 0.0053 \\
\hline cg15726387 & AluSz6 & -0.044 & 0.0950 & GSE18123 & 225925_s_at & USP48 & 0.15 & $<0.0001$ \\
\hline cg06385000 & AluSc & -0.041 & 0.0216 & GSE18123 & 227434_at & WBSCR17 & -0.46 & 0.0392 \\
\hline cg06385000 & AluSc & -0.041 & 0.0216 & GSE28521_FC & ILMN_1701557 & WBSCR17 & -0.19 & 0.0402 \\
\hline cg06385000 & AluSc & -0.041 & 0.0216 & GSE28521_TC & ILMN_1701557 & WBSCR17 & -0.23 & 0.0485 \\
\hline cg03870862 & L1MB3 & -0.063 & 0.0318 & GSE59288 & 23144 & ZСЗНЗ & 0.27 & 0.0193 \\
\hline
\end{tabular}

\section{Sensitivity and specificity of unique LINE-1 and Alu signatures in ASD variants.}

To predict diagnosis of the genetically homogenous ASD by using LINE-1 and Alu methylation signatures, we subsequently conducted ROC curves analysis of these loci and other probes within unique DMRs to distinguish each homogenous ASD variant from non-ASD and ASD with non-specific variants. For ASD with 16.p11.2 del, AluY within XKR6 (cg21300361) exhibited high sensitivity and specificity (AUC $=0.905,95 \% \mathrm{Cl}=0.83-0.98)$ to distinguish ASD with 16.p11.2 del from non-ASD and ASD with CHD8 variants as shown in the ROC curves (Figure 8D). In addition, the ROC curves of AluSq within ZNF107 (cg01772945) and L1MB3 within MYEOV2 (cg13749477) also exhibited high AUC value (AluSq: AUC = 0.900, $95 \% \mathrm{Cl}=0.83-0.97$ and L1MB3: AUC $=0.841,95 \% \mathrm{Cl}=0.74-0.95)$ (Figure $8 \mathrm{E}$ and $8 \mathrm{~F}$ ). In the ASD with CHD8 variants, LINE-1 
and Alu methylation signatures within candidate DMGs showed moderate sensitivity and specificity as demonstrated by AUC values (AUC range: 0.712-0.819) compared with the specificity of unique loci in ASD with 16.p11.2 del, including L1MC5 (cg22706070) within EHMT2 (Figure 9D), AluJo (cg06421197) within CASP1 (Figure 9E), and AluSx (cg18699242, cg01963623, cg02169692) within USP18 (Figure 9F). Our findings suggest that these novel DMPs at the LINE-1 and Alu elements could be used for clinical purposes. However, an independent cohort is required for validation, as we were limited by the percentage of ASD individuals affected by these genetic variants.

\section{Discussion}

Epigenetic modification is an important mechanism linking environmental and genetic factors, especially during the developmental process. Epigenetic age is a biomarker for age-related epigenetic changes as well as disease-specific (31), but it is unknown whether it contributes to ASD pathogenesis. DNAm age acceleration rates were significantly increased in the blood of mid-childhood ASD (5-11 years) using the method established by Wu et al (32). In this study, DNAm Age was calculated using Horvath's epigenetic clock algorithm, which shows a high correlation between the chronological age and DNAm Age. Interestingly, we discovered DNAm Age in ASD older than 11 years old was significantly decelerated but not in younger patients aged $<11$ years in our study. This observation is assumed to be related to the external exposures accumulated during childhood leading to developmental delay. Concomitantly, the mean of age acceleration difference in adolescence ASD (12-18 years old) was lower than their unaffected siblings (not statistically significant) (32). Additionally, we looked into the LINE-1 and Alu methylation profiles for ASD with these groups. Although DNAm Age was reduced in ASD individuals over the age of 11, the DNAm profile of the LINE-1 and Alu elements was significantly altered only in ASD under the age of 11 (see Additional file 9). These findings suggest that the DNAm signature of LINE-1 and Alu elements may change early which corresponds to early developmental and behavioral delays or deviancies. The epigenetic delays were also observed in post-mortem brain tissues of children with ASD (aged 5-8 years) (33).

In the heterogeneous ASD, we found no difference in global methylation (LINE-1 and Alu) (Figure 2A), which is consistent with our previous studies using lymphoblastoid cell lines that found no difference when all ASD were combined. In addition to Shpyleva's study, total methylation of LINE-1 in the ASD brain was also not significantly altered (25). The possibility is that the aberration of the global methylation levels (LINE-1 and A/u) may rely on family-specific REs or restrict to specific locations. Reducing the heterogeneity of ASD by classifying ASD based on clinical phenotype may be beneficial, as demonstrated by previous findings from our investigators $(21,26,34-36)$. Subcategorizing ASD allowed us to observe the hypomethylation of global methylation in ASD with $\mathrm{CHD} 8$ variants.

The aberration of LINE-1 or Alu elements during development may cause double-strand DNA breaks and DNA damage leading to the process of neurodegeneration $(37,38)$. Furthermore, identification of LINE-1 and Alu subfamilies has led to a better understanding of the association between the REs and human diseases because some subfamilies of LINE-1 and Alu elements remain active (20). To the best of our knowledge, our study is the first to identify the locus-specific methylation at LINE-1 and Alu elements in a subfamily-specific manner of the ASD blood samples. In this study, 7,165 DMPs at LINE-1 and Alu elements were identified in the heterogeneous ASD compared with non-ASD and the most of the DMPs were notably hypermethylated. We observed these hypermethylated loci mapped to L1P, L1H, AluJ, and AluS elements, which are intermediate and youngest LINE-1, and oldest and intermediate $A / u$, respectively. This implies that hypermethylation suppressed the most active LINE-1 and Alu subfamilies (intermediate and young REs) in the heterogeneous ASD. However, the hypomethylation of intermediate and young of LINE-1 and Alu was shown in ASD with CHD 8 variants. Both hypermethylation and hypomethylation of REs interfere with gene expression of themselves and inserted genes in ASD $(21,25)$.

It is important to note that LINE-1 and Alu elements play important roles in human brain development and brain somatic mosaicism. LINE-1 and Alu elements can regulate nearby genes during brain development (39-42). LINE-1 and Alu retrotransposition occurred more frequently in the brain than germline cells (43). Furthermore, Coufal's study, which compared LINE-1 activity in fetal neural progenitor cells (NPCs) to other somatic cells, revealed that NPCs have high retrotransposition of LINE-1 (44). They also discovered low DNAm at the LINE-1 promoter as well as a high copy number of LINE-1 in brain

Page 16/34 
tissues when compared to other somatic cells $(44,45)$. Thus, we also performed an analysis in the validation cohort using data from post-mortem brain tissues (GSE131706), we identified significant 482 DMPs $(p<0.05)$ at LINE-1 and Alu elements. Interestingly, we found that all DMPs from the brain were overlapped with DMPs identified in the heterogenous ASD blood (see Additional file 10). These findings suggest that epigenetic dysregulation of LINE-1 and Alu elements in ASD may alter the function of autism-related genes regulated by these elements. To address this, we predicted the biological functions and networks of genes located nearby DMPs of LINE-1 and Alu elements. Neurological diseases and canonical pathways implicated in ASD were significantly associated with these genes (see Additional file 5). Moreover, interactome networks associated with ASD revealed several autism-related genes in the SFARI database (Figure 6). Especially, AluSg7 (cg16926147) within KCNQ3 gene and L1PA3 (cg24094412) within HCN1 gene that were hypermethylated and inversely related to aberrant gene expression in the blood and post-mortem brain tissues of several ASD cohort studies. Hypomethylated DMPs were also discovered in the most active Alu family, AluY (cg08998414), which is located on the $U B E 2 H$ gene and has an inverse relationship with gene expression. KCNQ3 encodes a protein involved in neuronal excitability; people with a de novo variant of this gene experience ASD features, and some were diagnosed with ASD (46). HCN1 encodes a hyperpolarization-activated cation channel that is widely expressed in the brain regions (47). HCN1 mutation causes epileptic encephalopathy and this mutation is associated with intellectual disability and autistic traits (48). UBE2H encodes an E2 ubiquitin-conjugating enzyme family protein that is involved in the protein ubiquitination mechanism. Genetic association and screening studies have shown that this gene is present in ASD individuals. $(49,50)$. Another interesting result is hypomethylation in the HAL1 family which was found exclusively in the heterogeneous ASD. HAL1 or half-L1 encodes only ORF1p which enhances the efficiency of their transposition, but the origin, biological properties, and subfamilies have not been well studied (51). HAL1 subfamilies were also not well annotated in our data. However, this result warrants further research of their biological activity in the ASD.

Here, we discovered LINE-1 and Alu methylation signatures in these genetically homogeneous ASD (both 16p11.2 del and CHD8 variants). In the ASD with 16p11.2del, only locus-specific changes at LINE-1 and Alu elements were observed (Figure 4). We identified unique DMPs which target genes differentially expressed in the several ASD cohort studies including AluY within XKR6 (cg21300361), AluSq within ZNF107(cg01772945), and L1MB3 within MYEOV2 (cg13749477). These genes were genetic risk variants for ASD identified in genome-wide association study (GWAS), single nucleotide polymorphisms (SNPs), and copy number variation (CNV) studies (52-54). In the case of ASD with the CHD8 variants, we observed a widespread reduction of LINE-1 and Alu methylation levels in global methylation and the active LINE-1 and Alu families (L1P, $\mathrm{L} 1 \mathrm{H}$, and AluS). This change has far-reaching implications for even the oldest and fossil family (AluJ and FAM), as well as FRAM family. Furthermore, the unique LINE-1 and Alu methylation signatures of ASD with CHD8 variants, such as L1MC5 (cg22706070) within EHMT2, AluJo (cg06421197) within CASP1, and AluSx (cg18699242, cg01963623, cg02169692) within USP18, were also hypomethylated. However, we found that these alterations are inconsistent with the heterogeneous ASD profile, in which most DMPs were hypermethylated. These results may be caused by disease-specific genetic variants of $C H D 8$ that is a huge difference from the ASD without any genetic variants or with undefined ones. CHD8 is a chromatin remodeling/modifier factor that plays a role in the transcription process required for brain development (10). LINE-1 and Alu elements have an activation and a repressive chromatin mark that is bound by several chromatin remodeling/modifier factors $(39,42,55,56)$. Aberrant $C H D 8$ function may be leading to changes in genome-wide epigenetic marks which can affect a variety of gene regulatory mechanisms. The inverse relationship between LINE-1/Alu methylation and gene expression were also observed in the ASD with CHD8 variants. EHMT2, located nearby L1MC5, is a histone lysine methyltransferase involved with gene activation or repression. Gene and protein expression levels of EHMT2 were significantly increased in the post-mortem brain tissues of ASD $(57,58)$. CASP1 encodes cysteine-aspartic acid protease (caspase) enzyme involving apoptosis, monocyte cell fate, and immune response (59). CASP1, located nearby AluJo, was significantly elevated in the peripheral blood mononuclear cells of ASD (60), as well as overexpressed in two ASD studies including blood and post-mortem brain tissues. USP18 is a protein in the ubiquitin pathway which is essential for cell cycle, cell differentiation, and proliferation (61) and its CNV has been reported in ASD individuals (62). In transcriptome data obtained from several ASD studies, USP18 was significantly decreased, but not inversely related to hypomethylated positions of AluSx located in the upstream region of USP18 gene. However, three probes (cg18699242, cg01963623, and cg27281093)

Page $17 / 34$ 
at the same regions have been reported to be hypomethylated and they are the $C D H 8$ signature in the previous study (63). Our findings showed that DNAm of LINE-1 and Alu elements, located in the target genes, are connected with ASD-related genes. Moreover, biological functions and interactome of the genes located nearby LINE-1 and Alu methylation signatures in the ASD cohorts were associated with neurological diseases and developmental disorders, as well as canonical pathways implicated in ASD.

Unlike genetic changes, epigenetic alterations are not recorded in the genome and cannot be identified by genome sequencing. DNAm signatures are identified by comparing the methylation patterns of affected individuals to those typically developing control individuals. Several DNAm signatures have been established, and their effectiveness demonstrated as epigenetic markers for identifying variations of uncertain significance as pathogenic or benign (64). Although ASD pathogenesis occurs in the brain tissue, other systems such as the immune (65), metabolic (66), and gastrointestinal systems (67) are also affected in ASD individuals. DNAm in the blood is highly correlated to brain tissue samples and reflects environmental exposure (68). The discovery of distinct LINE-1 and Alu methylation signatures in ASD blood outlines their clinical potential to be used as non-invasive biomarkers. We conducted ROC curves analysis to predict a sensitivity and specificity of diagnosis with ASD using unique DMPs at LINE-1 and Alu elements identified in the blood of ASD individuals. Our findings show that LINE-1 and Alu methylation can be used to identify ASD with specific variants from unaffected individuals and classify them. However, additional research is required to determine its sensitivity and specificity in large and independent ASD cohorts.

In summary, we interrogated DNAm signatures of LINE-1 and Alu elements in the blood of ASD. DNAm signatures reflect environmental exposure linked to interaction with genetic factors through epigenetic processes. In the ASD cohort, DNAm signatures revealed changes in age acceleration residuals and LINE-1/Alu elements positions. The DNAm age was decelerated, meaning that an individual with ASD is epigenetically younger than their chronological age. Alteration of LINE-1 and Alu methylation induced a change in expression of genes located nearby these elements. Moreover, these genes have important functions related to neurodevelopment and cellular signaling. Disruption of these functions may lead to ASD features (Figure 10).

Because of the limitation of available post-mortem brain tissues for each ASD variant in publicly available datasets, our analyses were carried out using methylation data from ASD blood. Further research with a large number of post-mortem brain tissues is required. We did not perform multiple test corrections in the analysis of the validation cohort using post-mortem brain tissues, due to the small sample size which affects the statistical analysis power. Moreover, methylome and transcriptome datasets used in our study were obtained from different cohorts. However, transcriptome data from several ASD studies may reflect the heterogeneity of ASD, and one of the ASD cohorts in our analyses is a heterogeneous group. It is important to note that changes in LINE-1 and Alu methylation may occur as a result of a genetic factor in the genetically homogeneous ASD.

\section{Conclusions}

Locus-specific DNAm of LINE-1 and Alu elements in ASD, as well as its functional impact on gene regulation, were firstly reported in our study. Our analyses revealed LINE-1 and Alu methylation changes in a locus- and family-specific manner which were different according to each ASD cohort. By integrating methylome and transcriptome data, the target genes of LINE-1 and Alu elements were identified. These target genes were differentially expressed in multiple ASD cohorts, and their functions were related to neurological diseases and developmental disorders such as ASD. Our research also demonstrated that the LINE-1 and Alu signatures can be applied to diagnose and classify people with ASD. Finally, our finding will provide a better understanding of the impact of LINE-1 and Alu elements in ASD, at least in the blood. Our study provides evidence supporting future studies on the role of LINE-1 and Alu related to ASD neuropathology using human post-mortem brain tissues. However, further functional studies will be necessary to investigate the subsequent impact upon the target genes and fully elucidate the role of REs in ASD biology. 


\section{Abbreviations}

16p11.2del: 16p11.2 deletion

3'UTR: 3' untranslated region

5'UTR: 5' untranslated region

ASD: Autism spectrum disorder

CASP1: Caspase 1

CHD8: Chromodomain helicase DNA-binding protein 8

CNV: Copy number variation

DEGs: Differentially expressed genes

DMGs: Differentially methylated genes

DMPs: Differentially methylated positions

DMRs: Differentially methylated regions

DNAm: DNA methylation

EAA: Epigenetic age acceleration

EHMT2: Euchromatic Histone Lysine Methyltransferase 2

FDR: False discovery rate

FNBP1: Formin binding protein 1

GWAS: Genome-wide association study

HCN1: Hyperpolarization activated cyclic nucleotide gated potassium channel 1

KCNQ3: Potassium voltage-gated channel subfamily Q member 3

LINE-1: Long interspersed nucleotide element-1

MeCp2: Methyl-CpG binding protein 2

MYEOV2: Myeloma overexpressed 2

NDST1: N-deacetylase and N-sulfotransferase 1

ROC: Receiver operating characteristic

SNPs: Single nucleotide polymorphisms

TSS: Transcriptional start site

UBE2H: Ubiquitin Conjugating Enzyme E2 H

USP18: Ubiquitin-specific peptidase 18

Page 19/34 
USP6: Ubiquitin specific peptidase 6

XKR6: $X K$ related 6

ZNF107: Zinc Finger Protein 107

\section{Declarations}

\section{Ethics approval and consent to participate}

Not applicable

\section{Consent for publication}

Not applicable

\section{Availability of data and materials}

The datasets used and/or analyzed during the current study are available from the corresponding author on reasonable request.

\section{Competing interests}

The authors declare that they have no competing interests

\section{Funding}

This study was supported by Ratchadapisek Somphot Fund for Supporting Research Unit, Chulalongkorn University (grant number GRU 6300437001-1 and GRU_64_033_37_004) and the National Research University Project, Office of Higher Education Commission (NRU59-031-HR), awarded to Tewarit Sarachana), Postdoctoral Fellowship Program, Siriraj Hospital, Mahidol University (awarded to Chanachai Sae-Lee), and the Second Century Fund (C2F), Chulalongkorn University (awarded to Thanit Saeliw).

\section{Authors' contributions}

T.Sae. performed transcriptome analysis, biological function, and interactome prediction, and drafted the manuscript under the supervision of T.S., T.T. and C.S. C.S., N.I., D.G., and T.P. performed DNAm analysis for LINE-1 and Alu elements. T.S. supervised transcriptome analysis and interpretation. T.S. supervised the biological function and interactome prediction using IPA. C.S. had primary responsibility for the final content. All authors read and approved the final manuscript.

\section{Acknowledgements}

We would like to thank Dr Hyang-Min Byun and Dr Timothy M. Barrow for their assistance with the analysis of DNA methylation of REs.

\section{References}

1. Association AP. Diagnostic and Statistical Manual of Mental Disorders. 5th edition. 2013.

2. Maenner MJ, Shaw KA, Baio J, EdS, Washington A, Patrick M, et al. Prevalence of Autism Spectrum Disorder Among Children Aged 8 Years - Autism and Developmental Disabilities Monitoring Network, 11 Sites, United States, 2016. MMWR Surveill Summ. 2020;69(4):1-12.

3. Schaefer GB, Mendelsohn NJ. Genetics evaluation for the etiologic diagnosis of autism spectrum disorders. Genet Med. 2008;10(1):4-12. 
4. Bolte S, Girdler S, Marschik PB. The contribution of environmental exposure to the etiology of autism spectrum disorder. Cell Mol Life Sci. 2019;76(7):1275-97.

5. Stamou M, Streifel KM, Goines PE, Lein PJ. Neuronal connectivity as a convergent target of gene $x$ environment interactions that confer risk for Autism Spectrum Disorders. Neurotoxicol Teratol. 2013;36:3-16.

6. Tordjman S, Somogyi E, Coulon N, Kermarrec S, Cohen D, Bronsard G, et al. Gene x Environment interactions in autism spectrum disorders: role of epigenetic mechanisms. Front Psychiatry. 2014;5:53.

7. Folstein SE, Rosen-Sheidley B. Genetics of autism: complex aetiology for a heterogeneous disorder. Nat Rev Genet. 2001;2(12):943-55.

8. Merner N, Forgeot d'Arc B, Bell SC, Maussion G, Peng H, Gauthier J, et al. A de novo frameshift mutation in chromodomain helicase DNA-binding domain 8 (CHD8): A case report and literature review. Am J Med Genet A. 2016;170A(5):1225-35.

9. Weiss LA, Shen Y, Korn JM, Arking DE, Miller DT, Fossdal R, et al. Association between microdeletion and microduplication at 16p11.2 and autism. N Engl J Med. 2008;358(7):667-75.

10. Weissberg O, Elliott E. The Mechanisms of CHD8 in Neurodevelopment and Autism Spectrum Disorders. Genes (Basel). 2021;12(8).

11. Handy DE, Castro R, Loscalzo J. Epigenetic modifications: basic mechanisms and role in cardiovascular disease. Circulation. 2011;123(19):2145-56.

12. Zaidi SK, Young DW, Montecino M, Lian JB, Stein JL, van Wijnen AJ, et al. Architectural epigenetics: mitotic retention of mammalian transcriptional regulatory information. Mol Cell Biol. 2010;30(20):4758-66.

13. Edgar RD, Jones MJ, Meaney MJ, Turecki G, Kobor MS. BECon: a tool for interpreting DNA methylation findings from blood in the context of brain. Transl Psychiatry. 2017;7(8):e1187.

14. van de Haar HJ, Burgmans S, Jansen JF, van Osch MJ, van Buchem MA, Muller M, et al. Blood-Brain Barrier Leakage in Patients with Early Alzheimer Disease. Radiology. 2016;281(2):527-35.

15. Andrews SV, Sheppard B, Windham GC, Schieve LA, Schendel DE, Croen LA, et al. Case-control meta-analysis of blood DNA methylation and autism spectrum disorder. Mol Autism. 2018;9:40.

16. Garcia-Perez JL, Widmann TJ, Adams IR. The impact of transposable elements on mammalian development. Development. 2016;143(22):4101-14.

17. Britten RJ, Baron WF, Stout DB, Davidson EH. Sources and evolution of human Alu repeated sequences. Proc Natl Acad Sci U S A. 1988;85(13):4770-4.

18. Smit AF, Toth G, Riggs AD, Jurka J. Ancestral, mammalian-wide subfamilies of LINE-1 repetitive sequences. J Mol Biol. 1995;246(3):401-17.

19. Khan H, Smit A, Boissinot S. Molecular evolution and tempo of amplification of human LINE-1 retrotransposons since the origin of primates. Genome Res. 2006;16(1):78-87.

20. Mills RE, Bennett EA, Iskow RC, Devine SE. Which transposable elements are active in the human genome? Trends Genet. 2007;23(4):183-91.

21. Saeliw T, Tangsuwansri C, Thongkorn S, Chonchaiya W, Suphapeetiporn K, Mutirangura A, et al. Integrated genome-wide Alu methylation and transcriptome profiling analyses reveal novel epigenetic regulatory networks associated with autism spectrum disorder. Mol Autism. 2018;9:27.

22. Sae-Lee C, Biasi J, Robinson N, Barrow TM, Mathers JC, Koutsidis G, et al. DNA methylation patterns of LINE-1 and Alu for pre-symptomatic dementia in type 2 diabetes. PLoS One. 2020;15(6):e0234578.

23. Barrow TM, Wong Doo N, Milne RL, Giles GG, Willmore E, Strathdee G, et al. Analysis of retrotransposon subfamily DNA methylation reveals novel early epigenetic changes in chronic lymphocytic leukemia. Haematologica. 2021;106(1):98110. 
24. Jacob-Hirsch J, Eyal E, Knisbacher BA, Roth J, Cesarkas K, Dor C, et al. Whole-genome sequencing reveals principles of brain retrotransposition in neurodevelopmental disorders. Cell Res. 2018;28(2):187-203.

25. Shpyleva S, Melnyk S, Pavliv O, Pogribny I, Jill James S. Overexpression of LINE-1 Retrotransposons in Autism Brain. Mol Neurobiol. 2018;55(2):1740-9.

26. Tangsuwansri C, Saeliw T, Thongkorn S, Chonchaiya W, Suphapeetiporn K, Mutirangura A, et al. Investigation of epigenetic regulatory networks associated with autism spectrum disorder (ASD) by integrated global LINE-1 methylation and gene expression profiling analyses. PLoS One. 2018;13(7):e0201071.

27. Garcia-Ortiz MV, de la Torre-Aguilar MJ, Morales-Ruiz T, Gomez-Fernandez A, Flores-Rojas K, Gil-Campos M, et al. Analysis of Global and Local DNA Methylation Patterns in Blood Samples of Patients With Autism Spectrum Disorder. Front Pediatr. 2021;9:685310.

28. Horvath S. DNA methylation age of human tissues and cell types. Genome Biol. 2013;14(10):R115.

29. Srivastava S, Sahin M. Autism spectrum disorder and epileptic encephalopathy: common causes, many questions. J Neurodev Disord. 2017;9:23.

30. Bell L, Wittkowski A, Hare DJ. Movement Disorders and Syndromic Autism: A Systematic Review. J Autism Dev Disord. 2019;49(1):54-67.

31. Bell CG, Lowe R, Adams PD, Baccarelli AA, Beck S, Bell JT, et al. DNA methylation aging clocks: challenges and recommendations. Genome Biol. 2019;20(1):249.

32. Wu X, Chen W, Lin F, Huang Q, Zhong J, Gao H, et al. DNA methylation profile is a quantitative measure of biological aging in children. Aging (Albany NY). 2019;11(22):10031-51.

33. Corley MJ, Vargas-Maya N, Pang APS, Lum-Jones A, Li D, Khadka V, et al. Epigenetic Delay in the Neurodevelopmental Trajectory of DNA Methylation States in Autism Spectrum Disorders. Front Genet. 2019;10:907.

34. Hu VW, Sarachana T, Kim KS, Nguyen A, Kulkarni S, Steinberg ME, et al. Gene expression profiling differentiates autism case-controls and phenotypic variants of autism spectrum disorders: evidence for circadian rhythm dysfunction in severe autism. Autism Res. 2009;2(2):78-97.

35. Hu VW, Steinberg ME. Novel clustering of items from the Autism Diagnostic Interview-Revised to define phenotypes within autism spectrum disorders. Autism Res. 2009;2(2):67-77.

36. Lee EC, Hu VW. Phenotypic Subtyping and Re-Analysis of Existing Methylation Data from Autistic Probands in Simplex Families Reveal ASD Subtype-Associated Differentially Methylated Genes and Biological Functions. Int J Mol Sci. 2020;21(18).

37. Frappart PO, McKinnon PJ. Mouse models of DNA double-strand break repair and neurological disease. DNA Repair (Amst). 2008;7(7):1051-60.

38. Pornthanakasem W, Kongruttanachok N, Phuangphairoj C, Suyarnsestakorn C, Sanghangthum T, Oonsiri S, et al. LINE-1 methylation status of endogenous DNA double-strand breaks. Nucleic Acids Res. 2008;36(11):3667-75.

39. Jonsson ME, Ludvik Brattas P, Gustafsson C, Petri R, Yudovich D, Pircs K, et al. Activation of neuronal genes via LINE-1 elements upon global DNA demethylation in human neural progenitors. Nat Commun. 2019;10(1):3182.

40. Polak P, Domany E. Alu elements contain many binding sites for transcription factors and may play a role in regulation of developmental processes. BMC Genomics. 2006;7:133.

41. Batzer MA, Deininger PL. Alu repeats and human genomic diversity. Nat Rev Genet. 2002;3(5):370-9.

42. Su M, Han D, Boyd-Kirkup J, Yu X, Han JJ. Evolution of Alu elements toward enhancers. Cell Rep. 2014;7(2):376-85.

43. Upton KR, Baillie JK, Faulkner GJ. Is somatic retrotransposition a parasitic or symbiotic phenomenon? Mob Genet Elements. 2011;1(4):279-82.

44. Coufal NG, Garcia-Perez JL, Peng GE, Yeo GW, Mu Y, Lovci MT, et al. L1 retrotransposition in human neural progenitor cells. Nature. 2009;460(7259):1127-31.

45. Muotri AR. L1 Retrotransposition in Neural Progenitor Cells. Methods Mol Biol. 2016;1400:157-63.

Page 22/34 
46. Sands TT, Miceli F, Lesca G, Beck AE, Sadleir LG, Arrington DK, et al. Autism and developmental disability caused by KCNQ3 gain-of-function variants. Ann Neurol. 2019;86(2):181-92.

47. Chang X, Wang J, Jiang H, Shi L, Xie J. Hyperpolarization-Activated Cyclic Nucleotide-Gated Channels: An Emerging Role in Neurodegenerative Diseases. Front Mol Neurosci. 2019;12:141.

48. Nava C, Dalle C, Rastetter A, Striano P, de Kovel CG, Nabbout R, et al. De novo mutations in HCN1 cause early infantile epileptic encephalopathy. Nat Genet. 2014;46(6):640-5.

49. Martin I, Vourc'h P, Mahe M, Thepault RA, Antar C, Vedrine S, et al. Association study of the ubiquitin conjugating enzyme gene UBE2H in sporadic ALS. Amyotroph Lateral Scler. 2009;10(5-6):432-5.

50. Autism Spectrum Disorders Working Group of The Psychiatric Genomics C. Meta-analysis of GWAS of over 16,000 individuals with autism spectrum disorder highlights a novel locus at 10q24.32 and a significant overlap with schizophrenia. Mol Autism. 2017;8:21.

51. Bao W, Jurka J. Origin and evolution of LINE-1 derived "half-L1" retrotransposons (HAL1). Gene. 2010;465(1-2):9-16.

52. Thomas TR, Koomar T, Casten L, Tener AJ, Bahl E, Michaelson JJ. Clinical autism subscales have common genetic liability that is heritable, pleiotropic, and generalizable to the general population. medRxiv. 2021.

53. Yingjun X, Haiming Y, Mingbang W, Liangying Z, Jiaxiu Z, Bing S, et al. Copy number variations independently induce autism spectrum disorder. Biosci Rep. 2017;37(4).

54. Grove J, Ripke S, Als TD, Mattheisen M, Walters RK, Won H, et al. Identification of common genetic risk variants for autism spectrum disorder. Nat Genet. 2019;51(3):431-44.

55. Ferrari R, de Llobet Cucalon LI, Di Vona C, Le Dilly F, Vidal E, Lioutas A, et al. TFIIIC Binding to Alu Elements Controls Gene Expression via Chromatin Looping and Histone Acetylation. Mol Cell. 2020;77(3):475-87 e11.

56. He J, Fu X, Zhang M, He F, Li W, Abdul MM, et al. Transposable elements are regulated by context-specific patterns of chromatin marks in mouse embryonic stem cells. Nat Commun. 2019;10(1):34.

57. Wang ZJ, Zhong P, Ma K, Seo JS, Yang F, Hu Z, et al. Amelioration of autism-like social deficits by targeting histone methyltransferases EHMT1/2 in Shank3-deficient mice. Mol Psychiatry. 2020;25(10):2517-33.

58. Balan S, Iwayama Y, Maekawa M, Toyota T, Ohnishi T, Toyoshima M, et al. Exon resequencing of H3K9 methyltransferase complex genes, EHMT1, EHTM2 and WIZ, in Japanese autism subjects. Mol Autism. 2014;5(1):49.

59. Denes A, Lopez-Castejon G, Brough D. Caspase-1: is IL-1 just the tip of the ICEberg? Cell Death Dis. 2012;3:e338.

60. Siniscalco D, Sapone A, Giordano C, Cirillo A, de Novellis V, de Magistris L, et al. The expression of caspases is enhanced in peripheral blood mononuclear cells of autism spectrum disorder patients. J Autism Dev Disord. 2012;42(7):1403-10.

61. Honke N, Shaabani N, Zhang DE, Hardt C, Lang KS. Multiple functions of USP18. Cell Death Dis. 2016;7(11):e2444.

62. Chen $\mathrm{CH}$, Chen $\mathrm{HI}$, Chien WH, Li LH, Wu YY, Chiu YN, et al. High resolution analysis of rare copy number variants in patients with autism spectrum disorder from Taiwan. Sci Rep. 2017;7(1):11919.

63. Siu MT, Butcher DT, Turinsky AL, Cytrynbaum C, Stavropoulos DJ, Walker S, et al. Functional DNA methylation signatures for autism spectrum disorder genomic risk loci: 16p11.2 deletions and CHD8 variants. Clin Epigenetics. 2019;11(1):103.

64. Chater-Diehl E, Goodman SJ, Cytrynbaum C, Turinsky AL, Choufani S, Weksberg R. Anatomy of DNA methylation signatures: Emerging insights and applications. Am J Hum Genet. 2021;108(8):1359-66.

65. Meltzer A, Van de Water J. The Role of the Immune System in Autism Spectrum Disorder. Neuropsychopharmacology. 2017;42(1):284-98.

66. Cheng N, Rho JM, Masino SA. Metabolic Dysfunction Underlying Autism Spectrum Disorder and Potential Treatment Approaches. Front Mol Neurosci. 2017;10:34.

67. Wasilewska J, Klukowski M. Gastrointestinal symptoms and autism spectrum disorder: links and risks - a possible new overlap syndrome. Pediatric Health Med Ther. 2015;6:153-66.

68. Tylee DS, Kawaguchi DM, Glatt SJ. On the outside, looking in: a review and evaluation of the comparability of blood and brain "-omes". Am J Med Genet B Neuropsychiatr Genet. 2013;162B(7):595-603. 
69. Barrett T, Troup DB, Wilhite SE, Ledoux P, Evangelista C, Kim IF, et al. NCBI GEO: archive for functional genomics data sets-10 years on. Nucleic Acids Res. 2011;39(Database issue):D1005-10.

70. Pelegi-Siso D, de Prado P, Ronkainen J, Bustamante M, Gonzalez JR. methylclock: a Bioconductor package to estimate DNA methylation age. Bioinformatics. 2021;37(12):1759-60.

71. WHO, https: //www.who.int/maternal_child_adolescent/adolescence/en/. Accessed 20 Dec 2021.

72. Fortin JP, Triche TJ, Jr., Hansen KD. Preprocessing, normalization and integration of the Illumina HumanMethylationEPIC array with minfi. Bioinformatics. 2017;33(4):558-60.

73. Benjamini Y, Drai D, Elmer G, Kafkafi N, Golani I. Controlling the false discovery rate in behavior genetics research. Behav Brain Res. 2001;125(1-2):279-84.

74. Saeed Al, Bhagabati NK, Braisted JC, Liang W, Sharov V, Howe EA, et al. TM4 microarray software suite. Methods Enzymol. 2006;411:134-93.

75. Jalili V, Afgan E, Gu Q, Clements D, Blankenberg D, Goecks J, et al. The Galaxy platform for accessible, reproducible and collaborative biomedical analyses: 2020 update. Nucleic Acids Res. 2020;48(W1):W395-W402.

76. Chen S, Zhou Y, Chen Y, Gu J. fastp: an ultra-fast all-in-one FASTQ preprocessor. Bioinformatics. 2018;34(17):i884-i90.

77. Kim D, Langmead B, Salzberg SL. HISAT: a fast spliced aligner with low memory requirements. Nat Methods. 2015;12(4):357-60.

78. Liao Y, Smyth GK, Shi W. featureCounts: an efficient general purpose program for assigning sequence reads to genomic features. Bioinformatics. 2014;30(7):923-30.

79. Love MI, Huber W, Anders S. Moderated estimation of fold change and dispersion for RNA-seq data with DESeq2. Genome Biol. 2014;15(12):550.

80. Kramer A, Green J, Pollard J, Jr., Tugendreich S. Causal analysis approaches in Ingenuity Pathway Analysis. Bioinformatics. 2014;30(4):523-30.

\section{Figures}


- Non-ASD

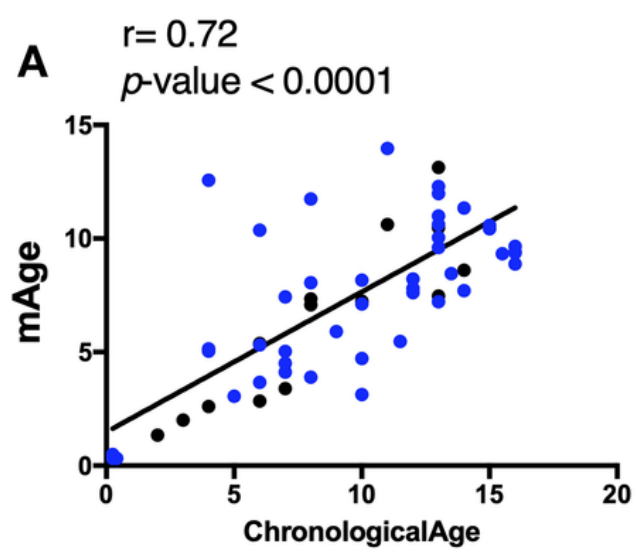

C

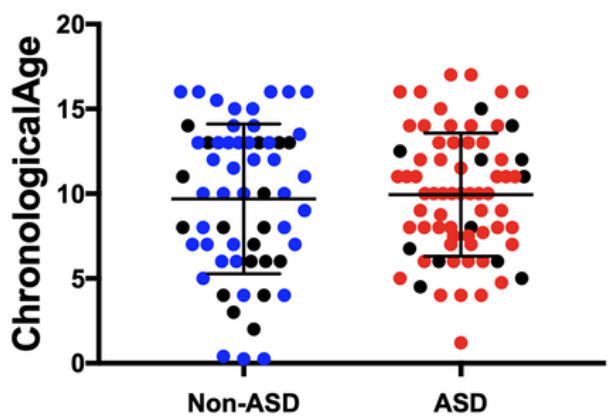

$E$

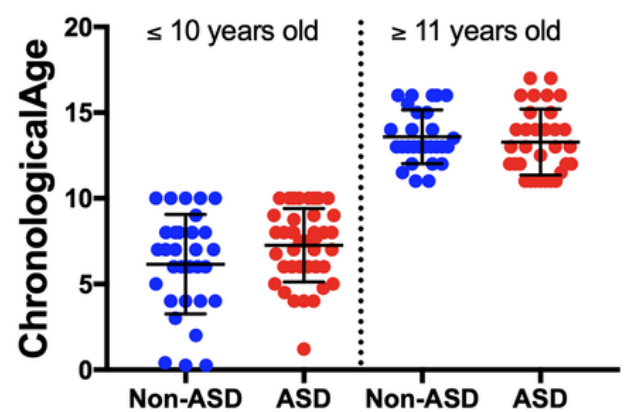

ASD

- Female

B $\quad \begin{aligned} & r=0.49 \\ & p \text {-value }<0.0001\end{aligned}$

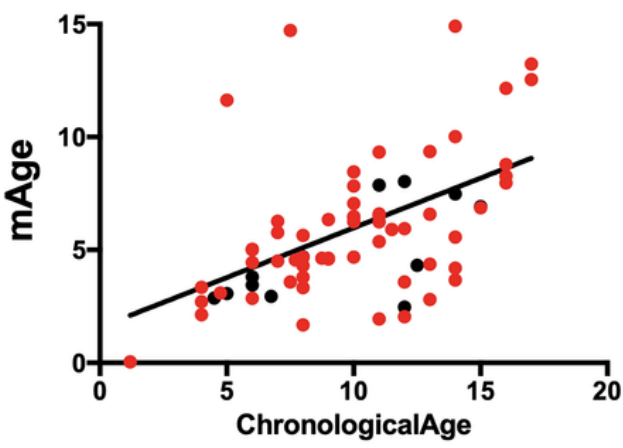

D

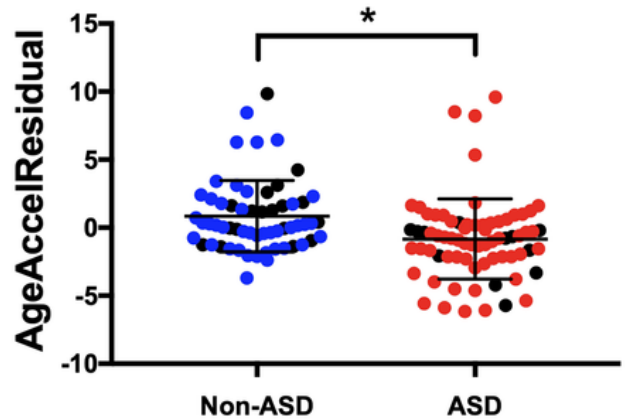

F

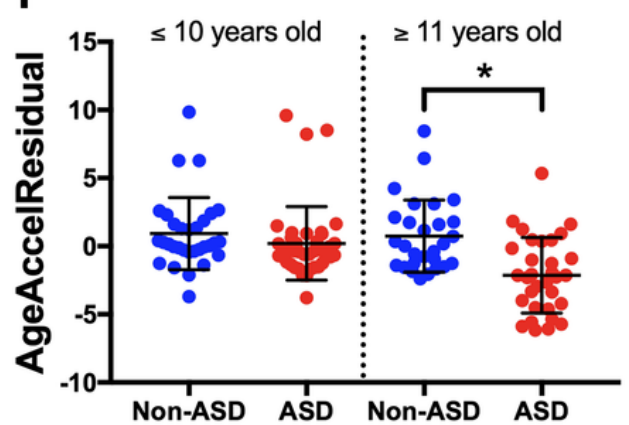

Figure 1

Age acceleration residual changes in ASD. Correlation of DNAm Age and chronological age in non-ASD $(n=59)(A)$ and ASD $(n=72)(B)$. Differences of the chronological age (C) and age acceleration residual (AgeAccelResidual) (D) between non-ASD and ASD. Differences of the chronological age (E) and AgeAccelResidual (F) between non-ASD and ASD by $\leq 10$ years old and $\geq 11$ years old. Mean \pm SD. ${ }^{*} p<0.05$. 

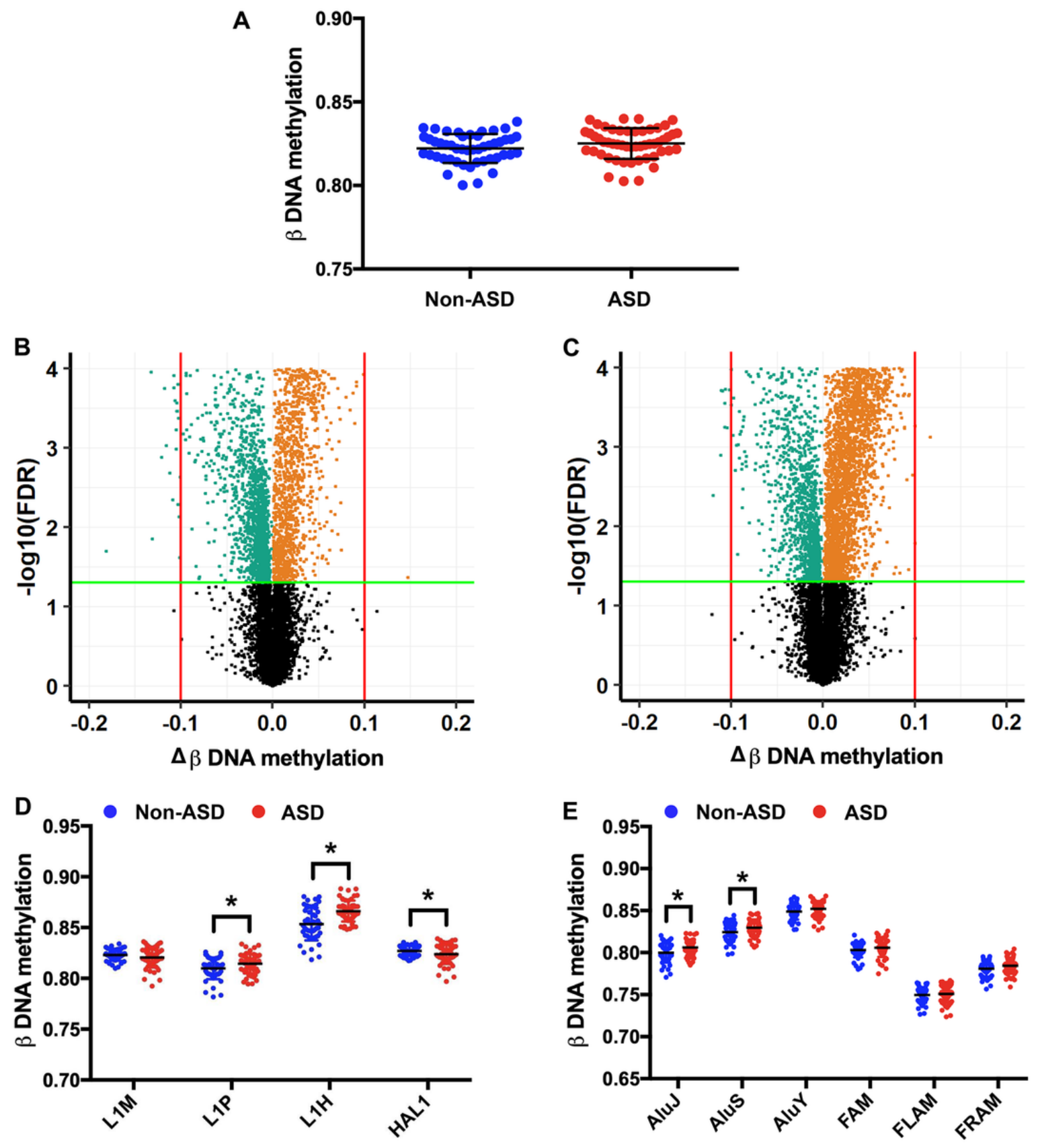

Figure 2

Methylation of repetitive elements (LINE-1 and Alu) in non-ASD $(n=48)$ and ASD $(n=52)$. Global DNA methylation $(A)$. Volcano plots of mean change in methylation $(\Delta \beta)$ of LINE-1 $(B)$ and $A / u(C)$ against -log10 FDR-adjusted p-value $\left(P_{F D R}\right)$ of ASD compared with non-ASD; the green line represents $P_{F D R}=0.05$, the red line represents $10 \%$ of methylation changes, green dots represent hypomethylation loci, and orange dots represent hypermethylation loci. Changes in DNA methylation $(\Delta \beta)$ of ASD compared with non-ASD by a subfamily of LINE-1 (D) and Alu (E). Mean \pm SD. ${ }^{*} p<0.05$. 


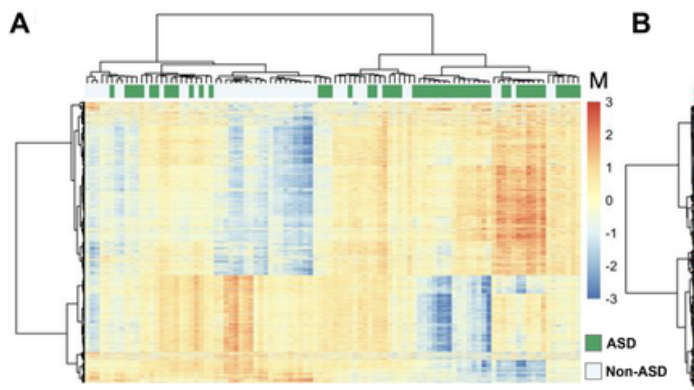

LINE-1 + Alu
B

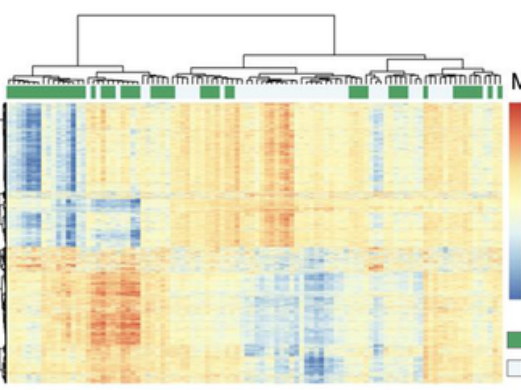

LINE-1

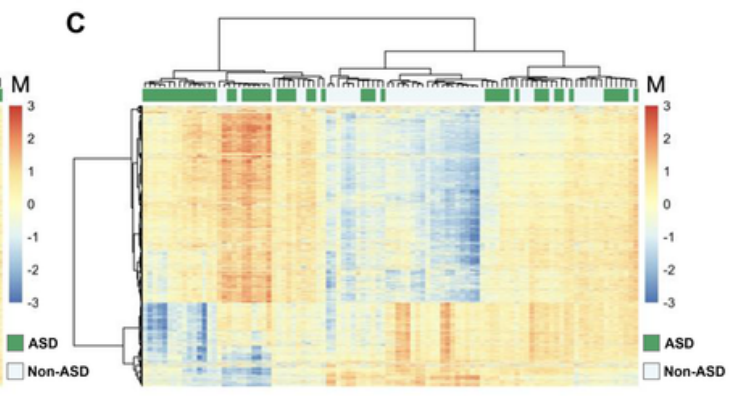

Alu

\section{Figure 3}

Unsupervised hierarchical cluster heatmap of the significant differentially methylated loci of the repetitive elements in nonASD and ASD. Clustering of the 7,165 significant differentially methylated loci (combining of LINE-1 and Alu loci) (A). Clustering of the 2,802 significant differentially methylated loci (LINE-1) (B). Clustering of the 4,363 significant differentially methylated loci $(A / U)(C)$. The color scale indicates methylation level (M value), from low (blue) to high (red). Green color represents ASD and white color represents non-ASD. 

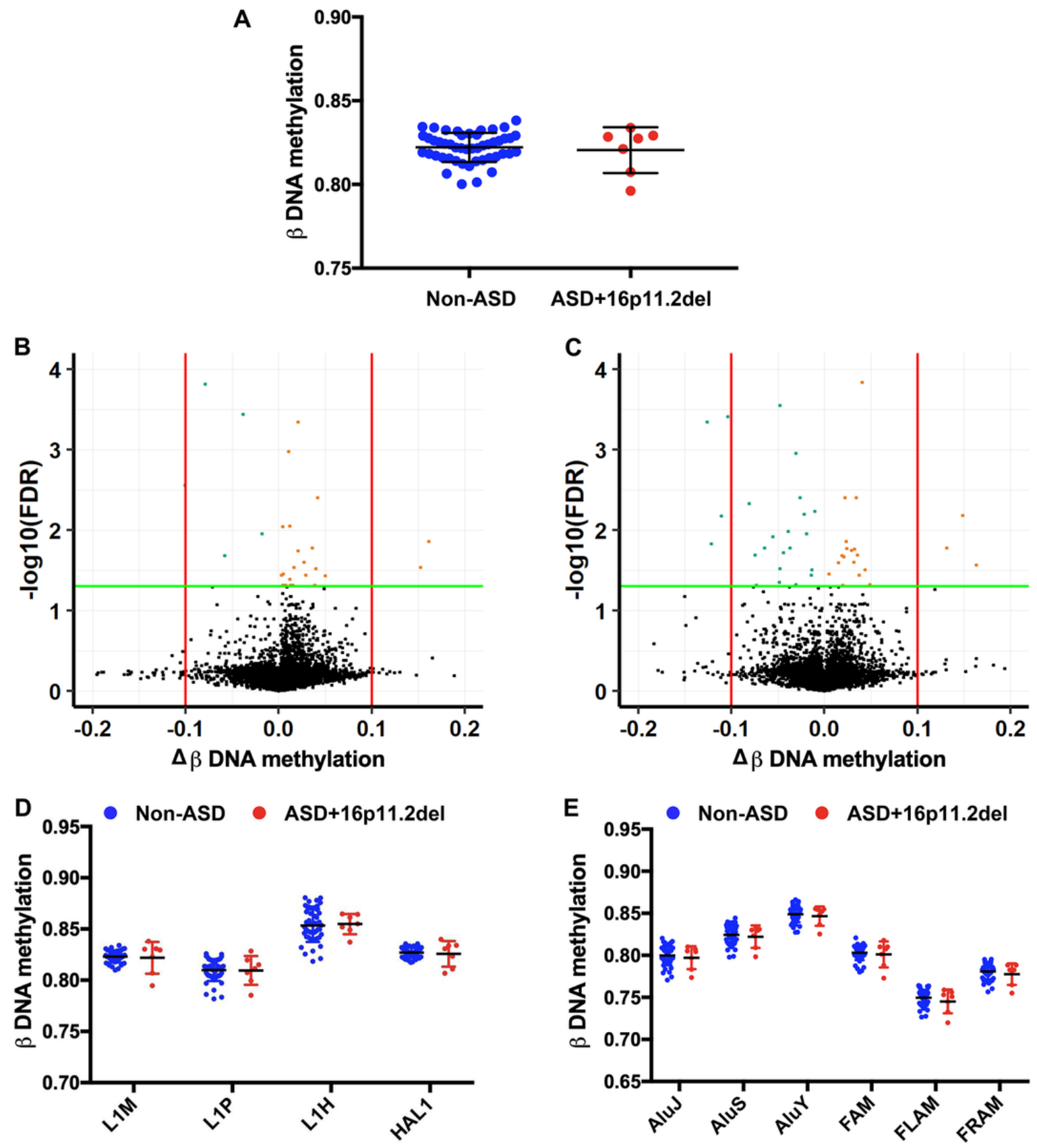

Figure 4

Methylation of repetitive elements (LINE-1 and Alu) in non-ASD $(n=48)$ and ASD patients who carry $16 p 11.2$ deletions $(n=$ 7). Global DNA methylation (A). Volcano plots of mean change in methylation ( $\Delta \beta$ ) of LINE-1 (B) and Alu (C) against -log10 FDR-adjusted $p$-value $\left(P_{F D R}\right)$ of ASD compared with non-ASD; the green line represents $P_{F D R}=0.05$, the red line represents $10 \%$ of methylation changes, green dots represent hypomethylation loci, and orange dots represent hypermethylation loci. Changes in DNA methylation $(\Delta \beta)$ of ASD with 16p11.2 deletion compared with non-ASD by a subfamily of LINE-1 (D) and Alu (E). Mean \pm SD. 
A
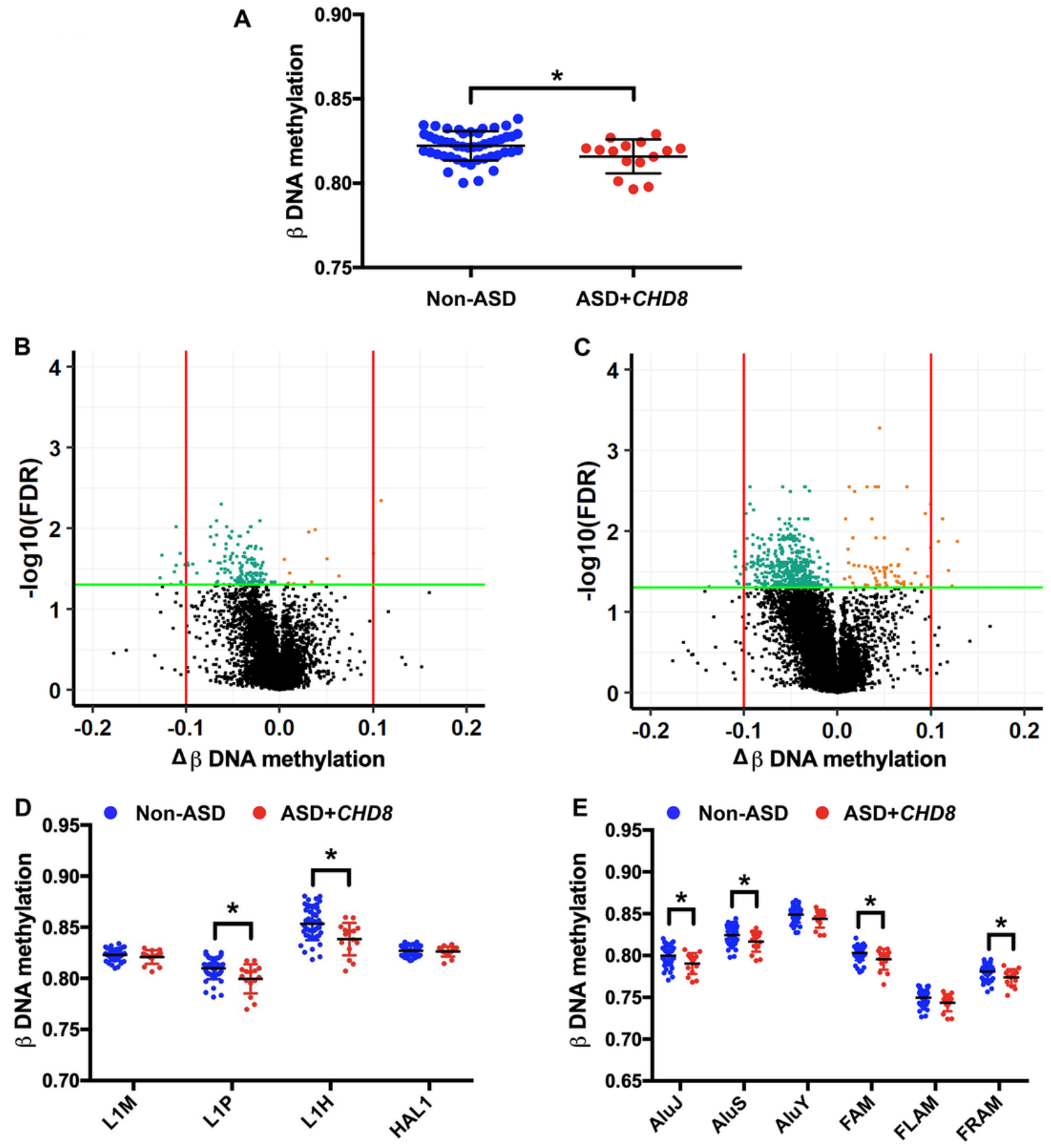

Figure 5

Methylation of repetitive elements (LINE-1 and A/u) in non-ASD $(n=48)$ and ASD patients who carry CHD 8 variants $(n=15)$. Global DNA methylation (A). Volcano plots of mean change in methylation $(\Delta \beta)$ of LINE-1 (B) and Alu (C) against -log10 FDRadjusted $p$-value $\left(P_{F D R}\right)$ of ASD compared with non-ASD; the green line represents $P_{F D R}=0.05$, the red line represents $10 \%$ of methylation changes, green dots represent hypomethylation loci, and orange dots represent hypermethylation loci. Changes in DNA methylation $(\Delta \beta)$ of ASD with $C H D 8$ compared with non-ASD by subfamily of LINE-1 (D) and Alu (E). Mean \pm SD. ${ }^{*} p<$ 0.05 . 


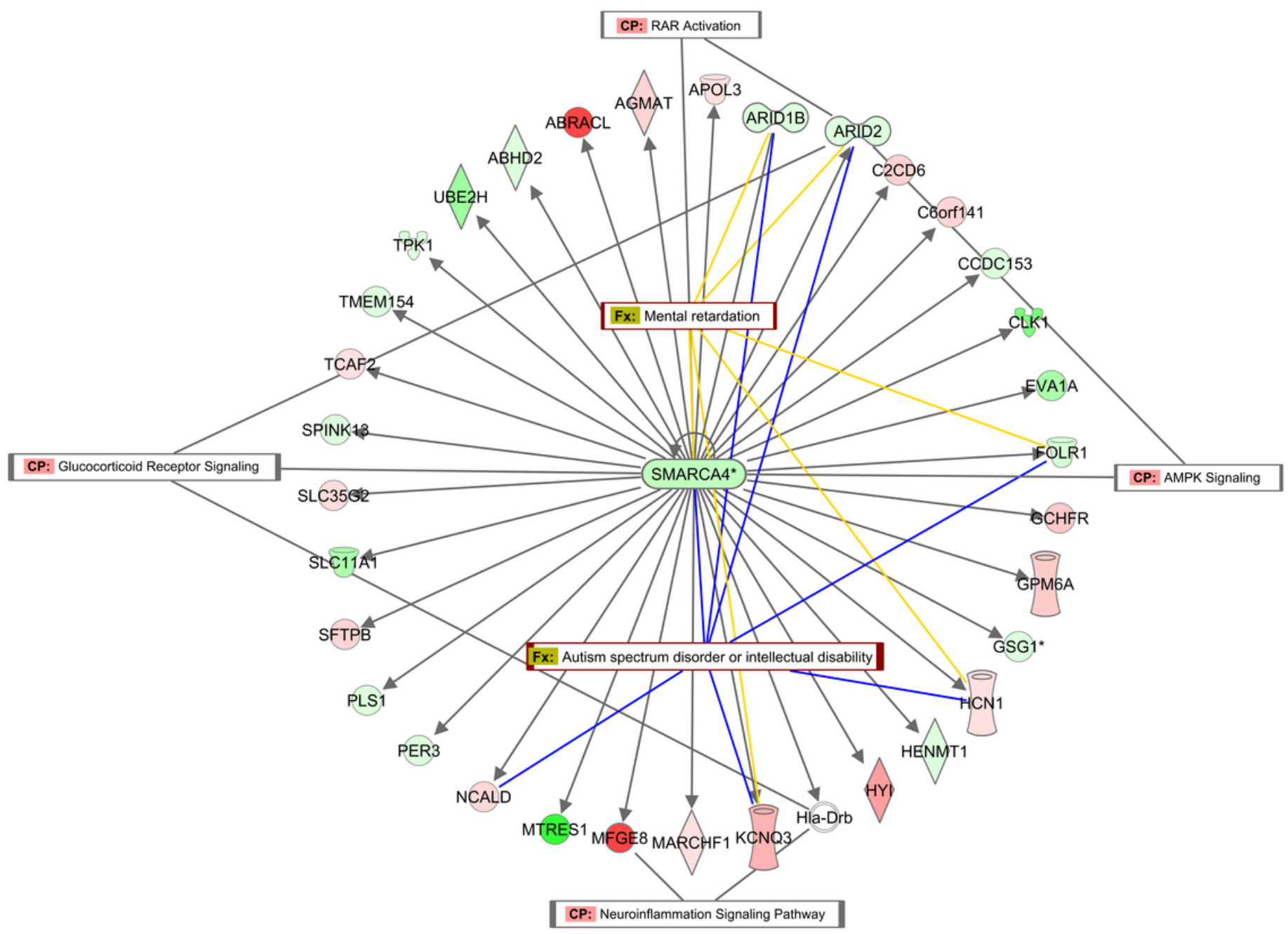

(ㄱ) 2000-2021 QIAGEN. All rights reserved.

\section{Figure 6}

The regulatory network of differentially methylated genes (DMGs) in heterogeneous ASD that is related to neurological diseases. The gene regulatory network was predicted by ingenuity pathway analysis software using the list of DMGs (colored; red $=$ hypermethylation; green = hypomethylation) 


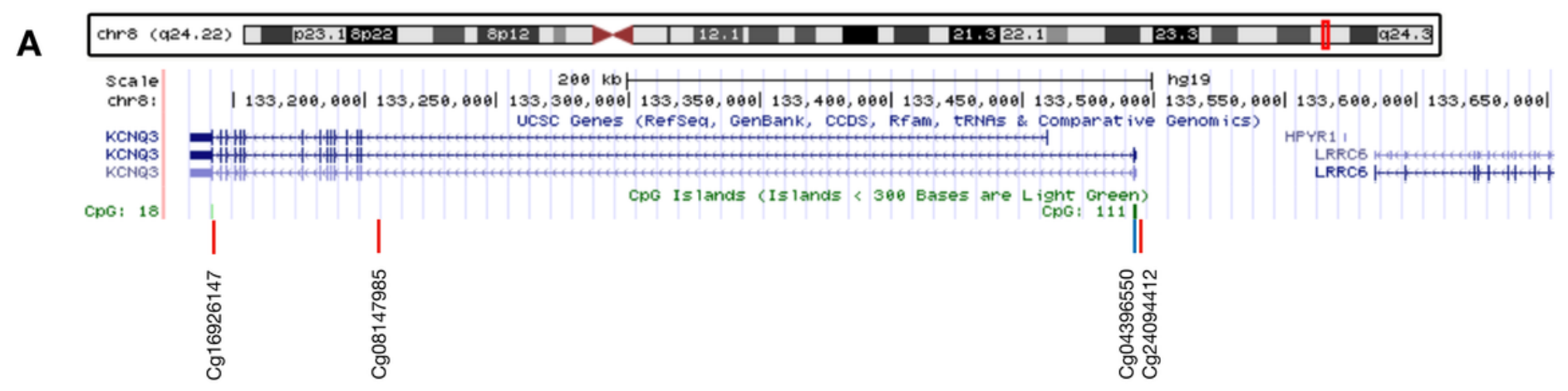

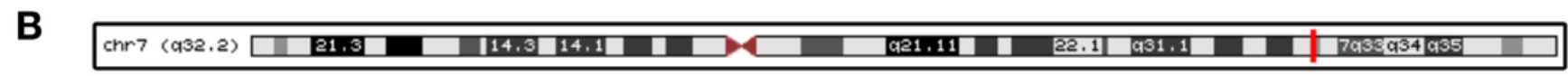
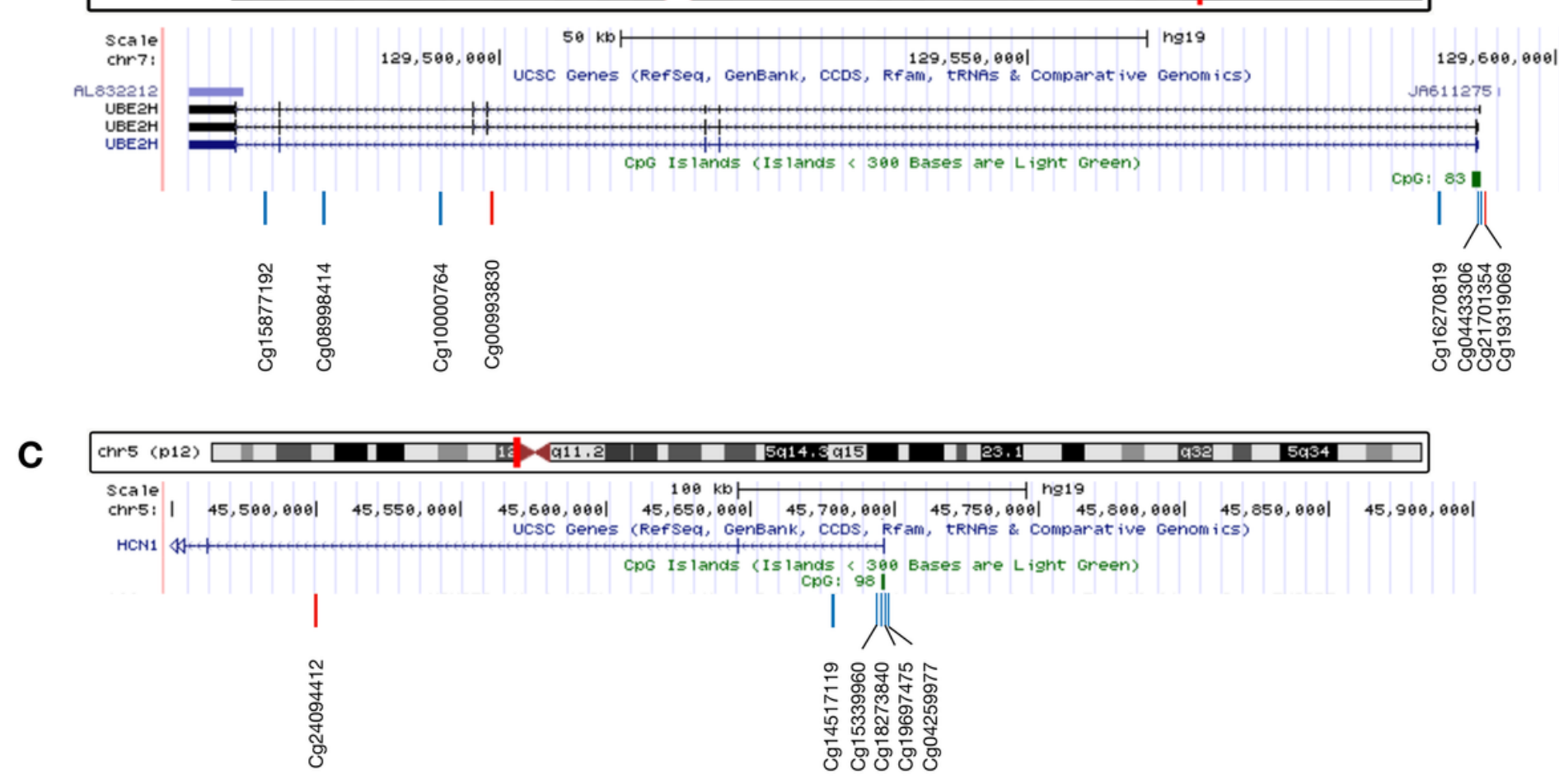

Figure 7

Genomic location of significant differentially methylated retrotransposons in heterogeneous ASD. Genomic location on potassium voltage-gated channel subfamily Q member 3 (KCNQ3) (A), ubiquitin conjugating enzyme E2 $H(U B E 2 H)(B)$, and hyperpolarization activated cyclic nucleotide gated potassium channel 1 (HCN1) (C): blue line represents hypomethylation, red line represents hypermethylation. 

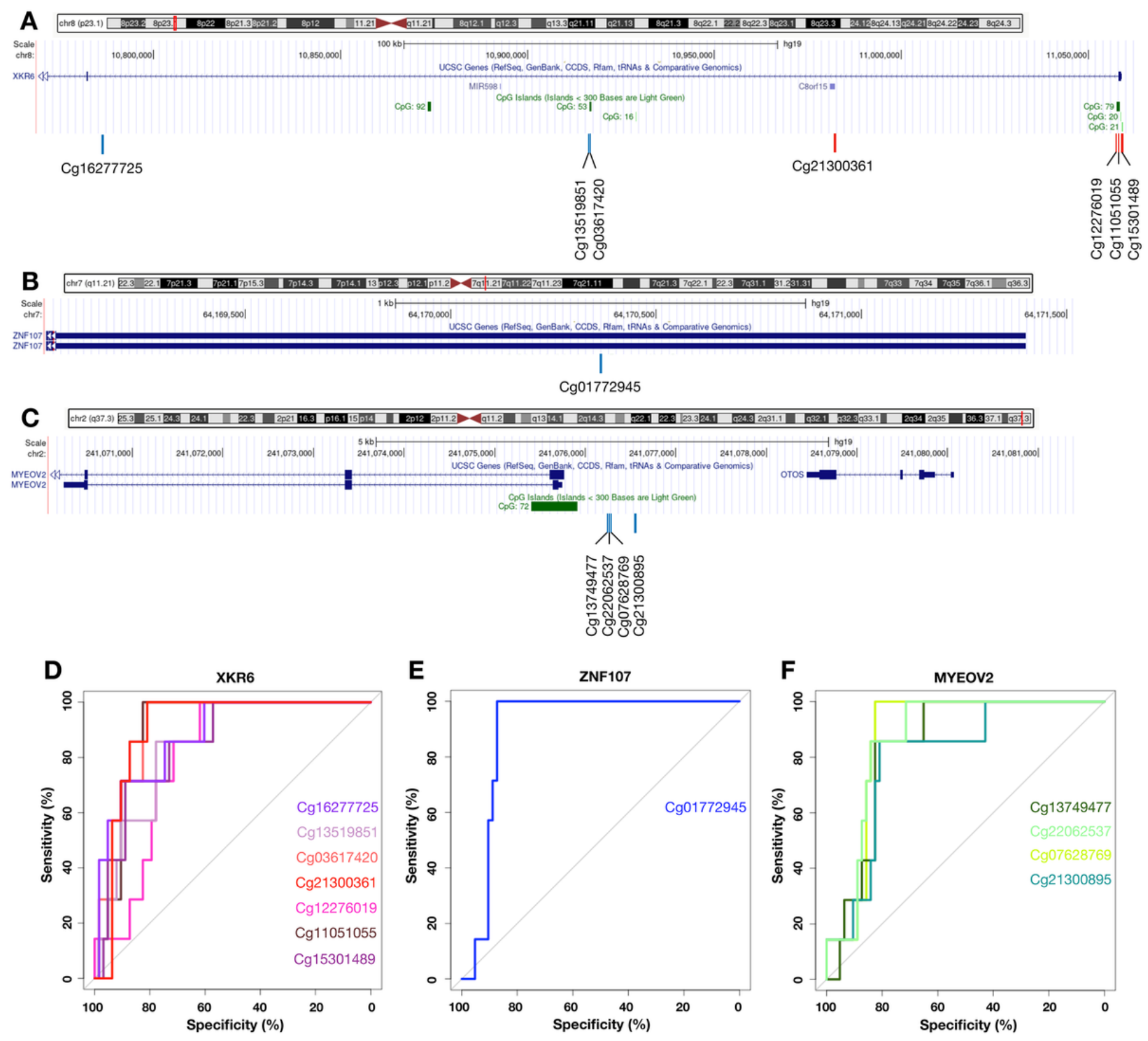

Figure 8

Genomic location and specificity of the unique differentially methylated regions in ASD with 16p11.2 deletion. Genomic location on XK related 6 (XKR6) (A), Zinc Finger Protein 107 (ZNF107) (B) and Myeloma overexpressed 2 (MYEOV2) (C): blue line represents hypomethylation, red line represents hypermethylation. The receiver operating characteristic (ROC) analysis of the unique differentially methylated regions of ASD with 16p11.2 deletion $(n=7)$ was performed against non-ASD $(n=48)$ and ASD with $C H D 8$ variants $(n=15)$. Specificity and sensitivity of the unique differentially methylated regions for $X K R 6$ (D), ZNF107 (E) and MYEOV2 (F). 

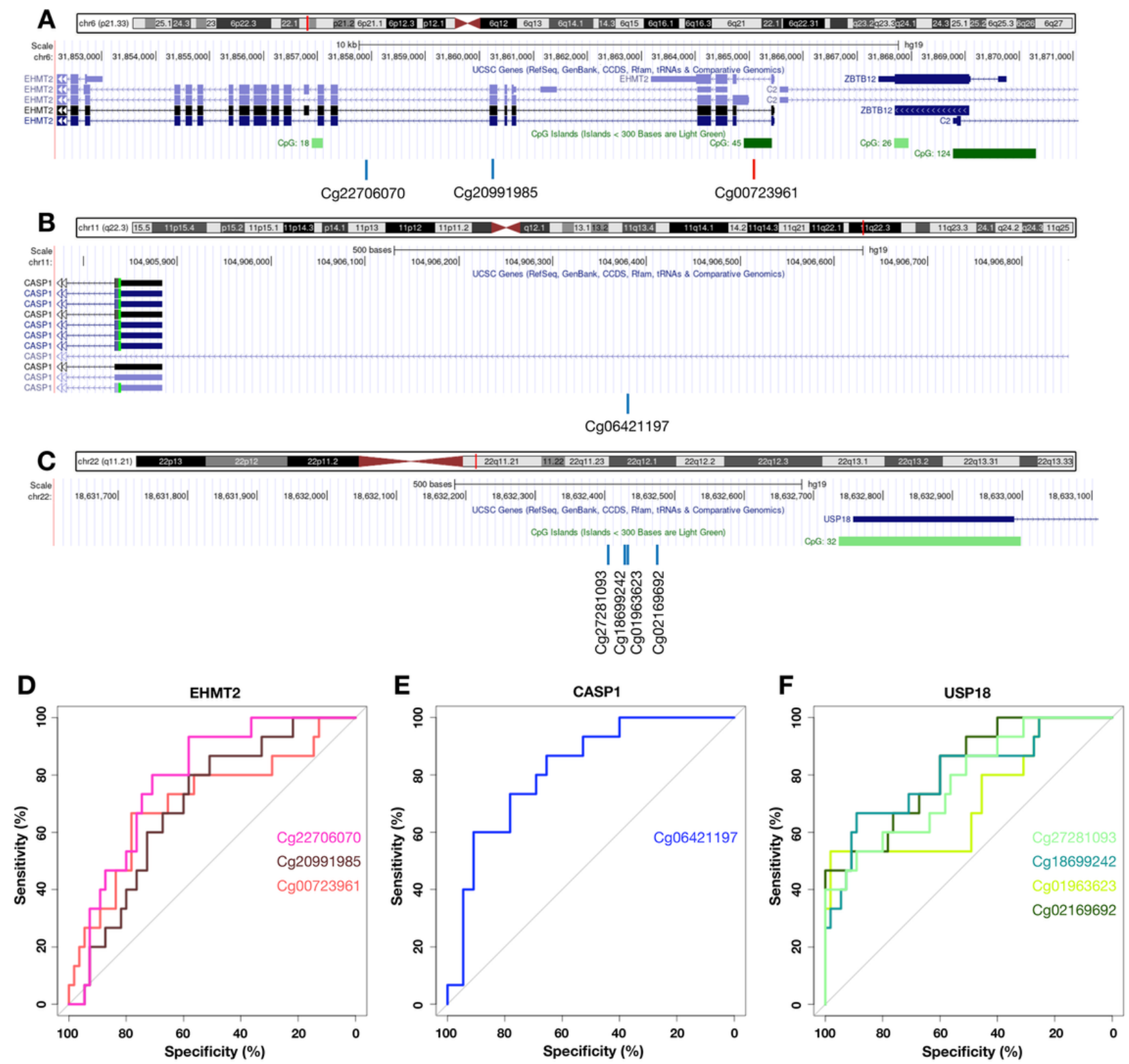

Figure 9

Genomic location and specificity of the unique differentially methylated regions in ASD with $C H D 8$ variants. Genomic location on Euchromatic Histone Lysine Methyltransferase 2 (EHMT2) (A), Caspase 1 (CASP1) (B) and Ubiquitin Specific Peptidase 18 (USP18) (C): blue line represents hypomethylation, the red line represents hypermethylation. The receiver operating characteristic (ROC) analysis of the unique differentially methylated regions of ASD with CHD8 variants $(n=15)$ was performed against non-ASD $(n=48)$ and ASD with 16p11.2 deletion $(n=7)$. Specificity and sensitivity of the unique differentially methylated regions for EHMT2 (D), CASP1 (E) and USP18 (F). 


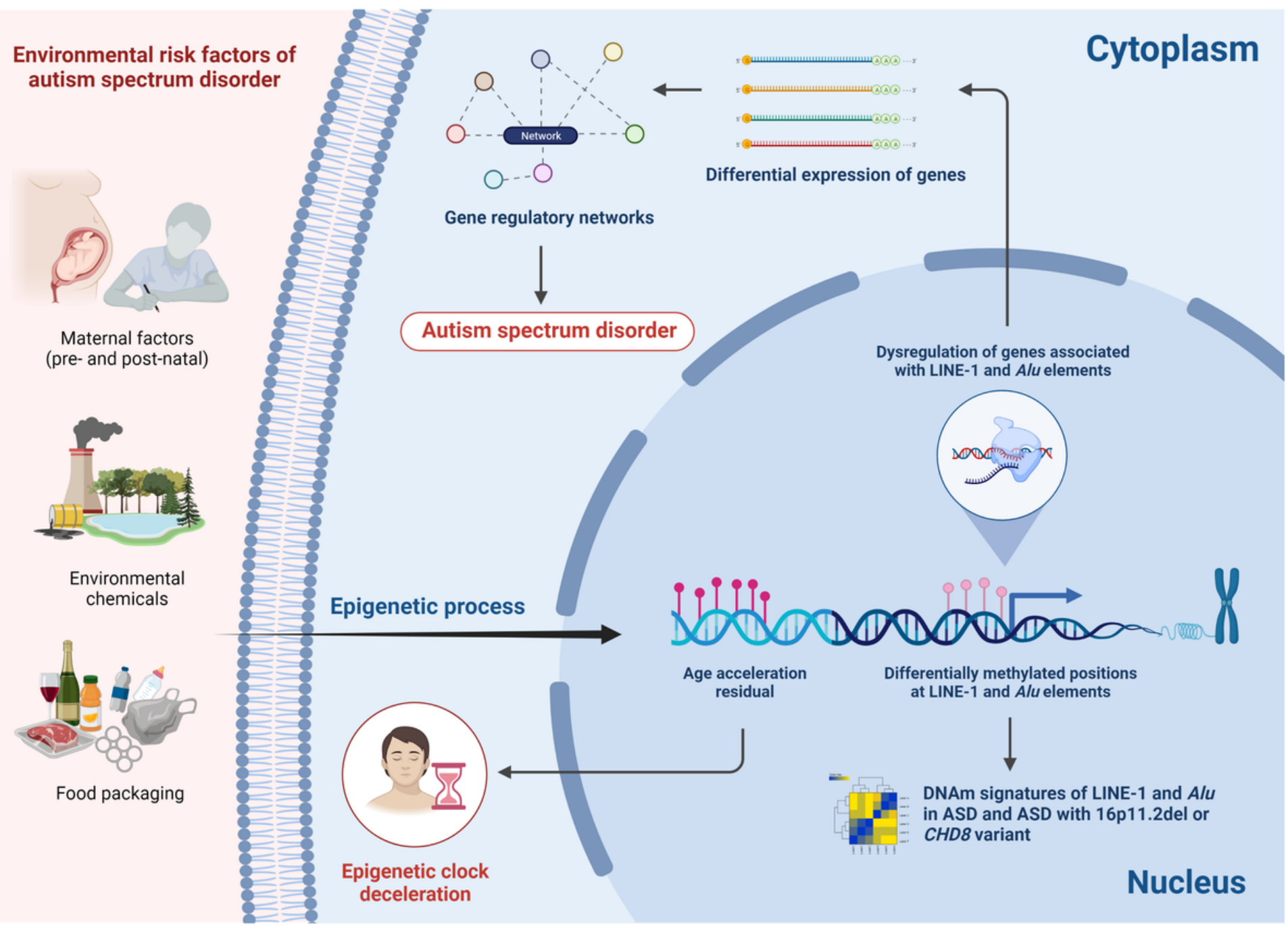

Figure 10

Schematic diagram illustrating a possible mechanism of LINE-1 and Alu elements in ASD.

\section{Supplementary Files}

This is a list of supplementary files associated with this preprint. Click to download.

- Additionalfile1.docx

- Additionalfile2.tif

- Additionalfile3.tif

- Additionalfile4.tif

- Additionalfile5.docx

- Additionalfile6.docx

- Additionalfile7.docx

- Additionalfile8.tif

- Additionalfile9.tif

- Additionalfile10.tif 